\title{
Momentum Injection by Supernovae in the Interstellar Medium
}

\author{
Chang-Goo Kim and Eve C. Ostriker \\ Department of Astrophysical Sciences, Princeton University, Princeton, NJ 08544, USA \\ cgkim@astro.princeton.edu, eco@astro.princeton.edu
}

\begin{abstract}
Supernova (SN) explosions deposit prodigious energy and momentum in their environments, with the former regulating multiphase thermal structure and the latter regulating turbulence and star formation rates in the interstellar medium (ISM). However, systematic studies quantifying the impact of SNe in realistic inhomogeneous ISM conditions have been lacking. Using three-dimensional hydrodynamic simulations, we investigate the dependence of radial momentum injection on both physical conditions (considering a range of mean density $n_{0}=0.1-100 \mathrm{~cm}^{-3}$ ) and numerical parameters. Our inhomogeneous simulations adopt two-phase background states that result from thermal instability in atomic gas. Although the SNR morphology becomes highly complex for inhomogeneous backgrounds, the radial momentum injection is remarkably insensitive to environmental details. For our two-phase simulations, the final momentum produced by a single SN is given by $2.8 \times 10^{5} M_{\odot} \mathrm{km} \mathrm{s}^{-1} n_{0}^{-0.17}$. This is only $5 \%$ less than the momentum injection for a homogeneous environment with the same mean density, and only $30 \%$ greater than the momentum at the time of shell formation. The maximum mass in hot gas is also quite insensitive to environmental inhomogeneity. Strong magnetic fields alter the hot gas mass at very late times, but the momentum injection remains the same. Initial experiments with multiple spatially-correlated SNe show a momentum per event nearly as large as single-SN cases. We also present a full numerical parameter study to assess convergence requirements. For convergence in the momentum and other quantities, we find that the numerical resolution $\Delta$ and the initial size of the SNR $r_{\text {init }}$ must satisfy $\Delta, r_{\text {init }}<r_{\text {sf }} / 3$, where the shell formation radius is given by $r_{\mathrm{sf}}=30 \mathrm{pc} n_{0}^{-0.46}$ for two-phase models (or $30 \%$ smaller for a homogeneous medium).
\end{abstract}

Subject headings: methods:numerical - supernovae: general - ISM: supernova remnants - ISM: kinematics and dynamics

\section{INTRODUCTION}

The importance of supernova (SN) blastwaves to the interstellar medium (ISM) has been appreciated for many decades (Cox \& Smith 1974, McKee \& Ostriker 1977). In recent years, it 
has become increasingly clear that accurate treatment of supernova remnant (SNR) evolution and other energy inputs from massive stars is crucial not just for detailed models of the ISM and star formation, but also for models of galaxy formation and evolution. SNe are believed to play the predominant role in creating the hot phase and shaping the large-scale structure of the multiphase ISM (e.g., Norman \& Ikeuchi 1989; de Avillez \& Breitschwerdt 2004; Hill et al. 2012; Hennebelle \& Iffrig 2014), in driving turbulence in diffuse gas (e.g., Rosen \& Bregman 1995, Mac Low \& Klessen 2004, Joung \& Mac Low 2006), and in regulating star formation rates by maintaining the largescale turbulent pressure that limits gravitational condensation (e.g., Ostriker \& Shetty 2011; Kim et al. 2011, 2013; Shetty \& Ostriker 2012). Particularly important, as we shall discuss below, is the momentum injected to the ISM over the lifetime of a SNR. The role of SNe in dense, starforming molecular clouds is less clear; due to the time delay before SNe occur, other forms of star formation "feedback" including protostellar outflows, the pressure of ionized gas in H II regions, forces from direct and dust-reprocessed radiation, and the pressure of shocked stellar winds may be of comparable or greater importance in driving turbulence and destroying massive molecular clouds (see Krumholz et al. 2014, Dobbs et al. 2013, and references therein). Through their roles in controlling star formation and launching galactic winds, SNe also regulate the internal structure of galaxies and the cosmic evolution of galactic populations, and recent numerical simulations have followed this with increasingly detailed treatments (e.g., Springel \& Hernquist 2003; Stinson et al. 2006; Guedes et al. 2011; Agertz et al. 2013; Aumer et al. 2013; Hopkins et al. 2012a, 2014).

The expansion of SNRs in a uniform medium has been extensively studied via spherically symmetric models and is well characterized by several familiar stages (e.g., Woltjer 1972; Draine 2011). The SN explosion event ejects material into interstellar space with typical kinetic energy of $\sim 10^{51} \mathrm{erg}$ and mass of $\sim 1-10 M_{\odot}$. The ejecta expand freely as long as the mass swept up by the forward shock is smaller than the ejecta mass (the free expansion stage). As the reverse shock heats up the interior, the hot gas temperature and pressure become very high, and expansion into the ambient medium proceeds with negligible radiative cooling. This is the well known phase of evolution analyzed by Sedov (1959) and Taylor (1950) (the ST stage). In the ST stage, the outer shock radius varies as $r_{\mathrm{snr}} \propto t^{2 / 5}$, and self-similar solutions describe the interior structure very well. As the temperature drops, radiative cooling becomes important, and a thin and dense shell is formed at the outer edge of the SNR where the temperature is lowest and the density is highest. After shell formation, a pressure-driven snowplow (PDS) stage occurs while the interior hot gas has non-negligible pressure (e.g., Cox 1972, McKee \& Ostriker 1977). After the interior pressure is exhausted, the shell continues to expand and sweep up ISM gas as a momentumconserving snowplow. One-dimensional, spherical hydrodynamic simulations of SNRs with radiative cooling confirm these main stages, also showing that simple treatments of the PDS stage (assuming adiabatic evolution of the hot interior) are inadequate (e.g., Chevalier \& Gardner 1974; Cioffi et al. 1988; Thornton et al. 1998; Blondin et al. 1998).

Although spherical models of SNR evolution in a uniform medium are well developed, direct application to the ISM is questionable since the ISM in reality is highly inhomogeneous. Without 
SNe, the neutral atomic ISM would consist of two distinct phases, with dense cold neutral medium (CNM) clouds surrounded by a diffuse warm neutral medium (WNM), as a result of thermal instability (Field 1965; Field et al. 1969; Wolfire et al. 1995a, 2003). Where the CNM collects into a large enough cloud that the interior is shielded from UV, it becomes molecular (see e.g. Sternberg et al. 2014, and references therein), and molecular clouds themselves are highly inhomogeneous because they are pervaded by highly supersonic turbulence (e.g. McKee \& Ostriker 2007). The addition of SN-driven shocks to the ISM makes its inhomogeneity even more extreme, producing a third phase of extremely tenuous gas (McKee \& Ostriker 1977; Cox 2005). Although observational estimates are difficult, the hot ISM is believed to fill a fraction $\lesssim 30 \%$ of the Milky Way disk's volume (e.g. Ferriere 1998; Ferrière 2001; Könyves et al. 2007), with the majority filled by WNM (and embedded cold clouds).

Since CNM clouds are two orders of magnitude denser than the intercloud WNM but the cold and warm mass fractions of the atomic ISM are comparable (Heiles \& Troland 2003), SNR expansion will be altered compared to spherical models. Understanding the effects of cloudy structure on SNR evolution requires multi-dimensional numerical simulations. As early efforts, effects of cloud compression and evaporation have been implicitly considered in spherical numerical simulations (e.g., Cowie et al. 1981; Wolff \& Durisen 1987). However, most multi-dimensional numerical simulations have focused on the detailed evolution and fate of individual shocked clouds (e.g., Klein et al. 1994; Mac Low et al. 1994; Fragile et al. 2005; Nakamura et al. 2006; Shin et al. 2008; Orlando et al. 2008; Johansson \& Ziegler 2013) rather than SNR evolution as a whole within the cloudy ISM.

The dearth of systematic studies of the SNR evolution in the multiphase ISM via direct numerical simulations has also had a serious impact on larger scale simulations. Without a model for blastwave propagation in an inhomogeneous medium during weakly radiative stages, the momentum injection from SN feedback is sometimes severely underestimated by considering just the momentum of the immediate ejecta (e.g., Agertz et al. 2013). When SN feedback is modeled by thermal energy injection, insufficient numerical resolution leads to the so-called "overcooling-problem" (e.g., Katz 1992, which artificially suppresses the dynamical impact of SN feedback to the surrounding medium. Although it has long been considered a serious problem in galaxy formation simulations, firm resolution requirements to avoid overcooling have not yet been quantified. Instead, many studies simply adopt a prescription in which cooling is artificially turned off for a period of time (e.g., Thacker \& Couchman 2000, Stinson et al. 2006, Scannapieco et al. 2012). However, this delayed-cooling prescription has the opposite problem of extending the ST phase to unrealistically long times. The method adopted in Stinson et al. (2006), widely used in subsequent studies (e.g., Guedes et al. 2011; Brook et al. 2012; Stinson et al. 2013), disables cooling for the merging time of McKee \& Ostriker (1977), which exceeds the shell formation time by an order of magnitude. This results in the substantial overestimate of the momentum injection and hot gas production by SNe. To address some of these issues, Keller et al. (2014) have recently developed a (two-phase) subgrid model to follow feedback effects from superbubbles, in which the proportions of hot and 
cold subgrid phases are controlled by thermal conduction, and both phases cool radiatively.

For the purposes of building analytic models of the ISM and star formation, and as inputs to subgrid feedback models in numerical simulations of galaxy formation, two important quantities are the total momentum injected by a SNR, and the mass of hot gas that it produces. The momentum, which is transferred to the warm and cold gas, determines the amplitude of turbulence in the volume-filling ISM, and also enters inversely in determining the large-scale star formation rate (e.g. Ostriker \& Shetty 2011; Kim et al. 2011). The hot gas produced by SNRs is important because it may be able to escape the galaxy's gravitational potential and drive a wind (e.g. Chevalier \& Clegg 1985; Strickland \& Stevens 2000). Although both quantities are important, momentum is more easily characterized as it is conserved at late times, whereas the mass of hot gas steadily declines once cooling has begun.

In this paper, we investigate the momentum injection to the multiphase ISM by SNe, using direct three dimensional hydrodynamic simulations with cooling. We shall show that the total momentum injection to an inhomogeneous medium is similar to that in a homogeneous medium, with both comparable to the radial momentum at the end of the ST stage. This is an order of magnitude larger than the initial momentum of the SN ejecta. We shall also provide firm numerical conditions for resolving the ST stage (thereby obtaining the correct momentum injection and early evolution of the hot medium) using standard finite-volume methods for hydrodynamics on a grid.

The remainder of the paper is organized as follows. In Section 2, we first summarize theoretical models of spherical SNR evolution. We provide analytic estimates of the shell formation time and key quantities (size, mass, velocity, momentum, temperature) of the SNR at this epoch. We then carry out three different sets of simulations, with numerical prescriptions as described in Section 3. We present results from three-dimensional, hydrodynamic simulations for single SNe in a uniform background in Section 4. We delineate the detailed evolution and compare numerical results to analytic models in Section 4.1, and provide a systematic parameter study to find numerical convergence conditions in Section 4.2. In Section 5, we perform a similar systematic study for single SNe in the two-phase ISM. An initial exploration of effects of multiple correlated SNe in the twophase ISM is presented in Section 6 ${ }^{1}$ Finally, in Section 7, we summarize our results and discuss their implications. Here, we also relate our work to other recent studies, and discuss physical limitations of our models and their potential impact on our conclusions. In the Appendix, we present results from magnetohydrodynamic models analogous to those of Section 4, with a range of background magnetic field strengths.

\footnotetext{
${ }^{1}$ Although for single SNe, the momentum-conserving stage is typically reached well before the SNR radius approaches the scale height of the disk, this is not necessarily true for multiple SNe that drive a superbubble. If a superbubble breaks out of the disk, then energy and mass will be vented to the halo rather than injecting momentum to the ISM. In this work, however, we do not consider disk stratification and breakout.
} 


\section{ANALYTIC THEORIES}

In this section, we briefly review analytic theories for the dynamics of radiative SNRs. Here we consider the simplest case of spherical expansion, for SN energy $E_{\mathrm{SN}}$ and ejecta mass $M_{\mathrm{ej}}$ in a uniform medium with density of $\rho_{0}$ (see Ostriker \& McKee 1988 for a more comprehensive review). In simple analytic models, each evolutionary stage of the SNR expansion can be approximated by a power law expansion of the SNR radius in time as $r_{\mathrm{snr}} \propto t^{\eta}$. The value of the power law exponent $\eta$ distinguishes four main stages of the expansion (e.g., Cioffi et al. 1988, Draine 2011): free expansion $(\eta=1)$, Sedov-Taylor $(\eta=2 / 5)$, pressure-driven snowplow $(\eta=2 / 7)$, and momentum-conserving snowplow $(\eta=1 / 4)$. At each successive stage, the exponent decreases as the available power to drive expansion declines.

In the initial stage of evolution, the mass of the ejecta dominates the mass of material that has been swept up from the circumstellar medium. Thus, the ejecta expands ballistically into the circumstellar medium with nearly constant velocity $v_{\mathrm{ej}}=\left(2 E_{\mathrm{SN}} / M_{\mathrm{ej}}\right)^{1 / 2}$ so that $r_{\mathrm{snr}} \propto t(\eta=1)$. The free expansion stage ends when the swept-up mass $M_{\mathrm{sw}}=(4 \pi / 3) \rho_{0} r_{\mathrm{snr}}^{3}$ becomes comparable to the ejecta mass. By equating $M_{\mathrm{ej}}=M_{\mathrm{sw}}$, we obtain the end of the free expansion stage as (Draine 2011)

$$
t_{\text {free }}=\frac{r_{\text {free }}}{v_{\text {ej }}}=464 \mathrm{yr}\left(\frac{M_{\mathrm{ej}}}{3 M_{\odot}}\right)^{5 / 6} E_{51}^{-1 / 2} n_{0}^{-1 / 2}
$$

for the SNR radius of

$$
r_{\text {free }}=\left(\frac{3 M_{\mathrm{ej}}}{4 \pi \rho_{0}}\right)^{1 / 3}=2.75 \mathrm{pc}\left(\frac{M_{\mathrm{ej}}}{3 M_{\odot}}\right)^{1 / 3} n_{0}^{-1 / 3} .
$$

Here, $E_{51} \equiv E_{\mathrm{SN}} / 10^{51} \mathrm{erg}$, and $n_{0} \equiv n_{H} / 1 \mathrm{~cm}^{-3}$ where the hydrogen number density of the ambient medium is $n_{H}=\rho_{0} /\left(1.4 m_{\mathrm{H}}\right)$ for $10 \%$ Helium abundance.

After the reverse shock heats up the ejecta, the evolution of the SNR for $t>t_{\text {free }}$ is well described by evolution of an idealized, point source explosion since the pressure of the SNR far exceeds the ambient medium pressure. To a good approximation, in this stage we can neglect the ejecta mass, energy losses, and the pressure of the ambient medium, and the solution depends only on $E_{\mathrm{SN}}$ and $\rho_{0}$; this defines the ST stage. Simple dimensional arguments imply that any length scale in the solution at a given time $t$ should be proportional to $\left(E_{\mathrm{SN}} t^{2} / \rho_{0}\right)^{1 / 5}$. The internal structure of the SNR in the ST stage is therefore given by a similarity solution with a similarity variable $\xi \equiv r /\left(E_{\mathrm{SN}} t^{2} / \rho_{0}\right)^{1 / 5}$. A detailed solution gives $\xi_{0}=1.15167$ at the shock radius, when the specific heat ratio is $\gamma=5 / 3$. We thus have the shock radius, shock velocity, and the immediate postshock temperature during the ST stage given by:

$$
\begin{gathered}
r_{\mathrm{ST}}=5.0 \mathrm{pc} E_{51}^{1 / 5} n_{0}^{-1 / 5} t_{3}^{2 / 5}, \\
v_{\mathrm{ST}}=\frac{2}{5} \frac{r_{\mathrm{ST}}}{t}=1.95 \times 10^{3} \mathrm{~km} \mathrm{~s}^{-1} E_{51}^{1 / 5} n_{0}^{-1 / 5} t_{3}^{-3 / 5},
\end{gathered}
$$




$$
T_{\mathrm{ST}}=\frac{3}{16} \frac{\mu v_{\mathrm{ST}}^{2}}{k_{\mathrm{B}}}=5.25 \times 10^{7} \mathrm{~K} E_{51}^{2 / 5} n_{0}^{-2 / 5} t_{3}^{-6 / 5},
$$

where $\mu$ is the mean mass per particles, and $t_{3} \equiv t / 10^{3}$ yr (e.g., Draine 2011).

When radiative energy losses are no longer negligible, the ST stage ends. Cooling is strongest immediately behind the shock where the density is the highest, and a cool dense shell bounding the SNR forms. The shell formation time can be defined as the time at which the first shocked gas parcel cools (e.g., Cox \& Anderson 1982, Cox 1986). For a volumetric cooling rate $n_{H} n_{e} \Lambda(T)$, the cooling time $t_{\text {cool }} \equiv e /|d e / d t|$, where $e$ is the internal energy, of the postshock gas is

$$
t_{\text {cool }}=\frac{2.3}{1.2} \frac{k T_{\mathrm{ST}}}{(\gamma+1) n_{0} \Lambda\left(T_{\mathrm{ST}}\right)} .
$$

A gas parcel shocked at $t_{s}$ cools down during $t_{\text {cool }}$ and forms a shell at $t_{\mathrm{sf}}=t_{s}+t_{\text {cool }}$. The time for first shell formation can be obtained by substituting for $T_{\mathrm{ST}}$ from equation (5) in equation (6) and then finding the time $t_{s}$ that minimizes $t_{\mathrm{sf}}$ :

$$
t_{\mathrm{sf}}=4.4 \times 10^{4} \text { yr } E_{51}^{0.22} n_{0}^{-0.55} .
$$

Here, we have used an approximate power-law cooling function $\Lambda(T)=C\left(T / 10^{6} \mathrm{~K}\right)^{-\alpha}$ with $C=$ $1.1 \times 10^{-22} \mathrm{erg} \mathrm{s}^{-1} \mathrm{~cm}^{3}$ and $\alpha=0.7$ that gives a fairly good fit for our adopted cooling function in a range of $10^{5} \mathrm{~K}<T<10^{7.5}$ (see Draine 2011). Note that alternative approximations for the cooling function with $\alpha=0.5-1.0$ change the exponents for energy and density only slightly, in the range 0.21 to 0.24 and -0.57 to -0.53 , respectively. At the time of shell formation, the outer radius, velocity, postshock temperature, and total swept-up mass are given by:

$$
\begin{gathered}
r_{\mathrm{sf}}=22.6 \mathrm{pc} E_{51}^{0.29} n_{0}^{-0.42}, \\
v_{\mathrm{sf}}=202 \mathrm{~km} \mathrm{~s}^{-1} E_{51}^{0.07} n_{0}^{0.13}, \\
T_{\mathrm{sf}}=5.67 \times 10^{5} \mathrm{~K} E_{51}^{0.13} n_{0}^{0.26}, \\
M_{\mathrm{sf}}=1680 M_{\odot} E_{51}^{0.87} n_{0}^{-0.26} .
\end{gathered}
$$

It is notable that the velocity, post-shock temperature, and total swept-up mass of the remnant at the time of shell formation are insensitive to the ambient density. Physically, this is simply because cooling becomes quite strong when the temperature falls below $\sim 10^{6} \mathrm{~K}$ and $\mathrm{C}$ and $\mathrm{O}$ acquire electrons that can be collisionally excited (e.g. Draine 2011); reaching this temperature (and the corresponding shock speed) essentially defines the radiative stage of a SNR (Spitzer 1978).

At shell formation, the shock stalls, and the hot gas in the outer portion of the SNR is rapidly compressed into a cold thin shell. After shell formation, the shell is still pushed outward by overpressured hot gas in the interior of the SNR. Under certain idealizations, this leads to a so-called pressure-driven snowplow (PDS) stage. The equation of the motion for the thin shell in the idealized PDS stage can be written as

$$
\frac{d\left(M_{\mathrm{sh}} v_{\mathrm{snr}}\right)}{d t}=4 \pi r_{\mathrm{snr}}^{2}\left(P_{\mathrm{hot}}-P_{0}\right),
$$


where $M_{\mathrm{sh}}$ is the mass of the shell and $v_{\mathrm{snr}}=d r_{\mathrm{snr}} / d t$ is the expansion velocity of the SNR. If the radiative cooling of the hot interior gas is negligible (i.e. only the gas immediately behind the shock is assumed to cool) and the mass of hot gas is assumed to be constant, the interior pressure would drop only from adiabatic expansion as $P_{\text {hot }} \propto r_{\mathrm{snr}}^{-3 \gamma}$. For $\gamma=5 / 3$, setting $M_{\mathrm{sh}}=(4 \pi / 3) r_{\mathrm{snr}}^{3} \rho_{0}$ and dropping $P_{0}$ on the right-hand side leads to a self-similar solution with $\eta=2 / 7$ (see Ostriker $\&$ McKee 1988 for possible variations of this equation with other terms). For the idealized PDS solution, the SNR interior is treated as uniform, with a mean pressure

$$
P_{\text {hot }}=P_{\text {hot }, \mathrm{sf}}\left(\frac{r_{\mathrm{snr}}}{r_{\mathrm{sf}}}\right)^{-5}=P_{\mathrm{hot}, \mathrm{sf}}\left(\frac{t}{t_{\mathrm{sf}}}\right)^{-10 / 7},
$$

with

$$
P_{\mathrm{hot}, \mathrm{sf}}=\frac{E_{\mathrm{th}, \mathrm{ST}}}{2 \pi r_{\mathrm{sf}}^{3}}=2.4 \times 10^{6} k_{\mathrm{B}} \mathrm{cm}^{-3} \mathrm{~K} E_{51}^{0.13} n_{0}^{1.26},
$$

where $E_{\mathrm{th}, \mathrm{ST}}=0.717 E_{\mathrm{SN}}$. Note that the mean pressure is about a half of the post-shock pressure.

The final classical stage of a SNR begins when the interior pressure $P_{\text {hot }}$ has decreased sufficiently that it no longer exceeds the pressure of the ambient medium $P_{0}$, making the right hand side of Equation 12 zero. This leads to the constant radial momentum and a self-similar expansion with $\eta=1 / 4$.

We now summarize the momentum injection to the ISM from each of the above SNR expansion stages. In the free expansion stage, all the momentum is contained in the ejecta, and the magnitude of the radial momentum is

$$
p_{\text {free }}=M_{\mathrm{ej}} v_{\mathrm{ej}}=1.73 \times 10^{4} M_{\odot} \mathrm{km} \mathrm{s}^{-1}\left(\frac{M_{\mathrm{ej}}}{3 M_{\odot}}\right)^{1 / 2} E_{51}^{1 / 2} .
$$

Since free expansion by definition assumes negligible interaction with the circumstellar and/or interstellar medium, the ambient medium acquires no momentum at this stage.

In the ST stage, the propagating strong shock heats and accelerates the ambient medium. Using the ST self-similar solutions, the total radial momentum of the shocked hot gas can be obtained as

$$
p_{\mathrm{ST}}=\int \rho v 4 \pi r^{2} d r=2.69 \rho_{0} v_{\mathrm{ST}} r_{\mathrm{ST}}^{3}=2.21 \times 10^{4} M_{\odot} \mathrm{km} \mathrm{s}^{-1} E_{51}^{4 / 5} n_{0}^{1 / 5} t_{3}^{3 / 5} .
$$

The momentum at the time of shell formation (the end of the ST stage) is obtained by substituting equation (7) in equation (16):

$$
p_{\mathrm{sf}}=2.17 \times 10^{5} M_{\odot} \mathrm{km} \mathrm{s}^{-1} E_{51}^{0.93} n_{0}^{-0.13} .
$$

We note that this is nearly linear in the SN energy, and is quite insensitive to the ambient density. This is because the momentum of the SNR during the ST stage is $p \propto E / v$, and the expansion velocity at the time of shell formation from equation $(9)$ is very insensitive to both total energy and ambient density, $v_{\mathrm{sf}} \sim 200 \mathrm{~km} \mathrm{~s}^{-1}$. 
In the PDS stage, the mass and radial momentum in the SNR are dominated by the shell. Under the assumption that the interior expands adiabatically (which is not in fact satisfied; see below), the momentum that would be acquired in an idealized PDS stage after $t_{\text {sf }}$ can be obtained by integrating Equation (12), leading to total momentum:

$$
p_{\mathrm{PDS}}=p_{\mathrm{sf}}\left[1+0.66 \int_{1}^{t_{*}} \frac{d t_{*}}{r_{*}^{3}}\right]=p_{\mathrm{sf}}\left[1+4.6\left(t_{*}^{1 / 7}-1\right)\right],
$$

where $t_{*} \equiv t / t_{\mathrm{sf}}$ and $r_{*} \equiv r_{\mathrm{snr}} / r_{\mathrm{sf}}$. Although equation (18) suggests only a very slow growth of momentum with time, the increase over $p_{\text {sf }}$ would in principle be substantial if the interior of the remnant remained hot for $t \gg t_{\mathrm{sf}}$.

However, the above idealized treatment of the post-shell-formation evolution does not agree with detailed time-dependent solutions, and momentum injection in practice increases only modestly subsequent to shell formation. As we shall show in the following sections (see also Cioffi et al. 1988), the classical PDS assumption of a hot, adiabatic interior with no mass loss does not apply. In fact, the mass of hot gas interior to the shell steadily decreases in time, because the outermost part flows into the shell and cools. Since the pressure decreases more rapidly than the simple adiabatic expectation, there is only a brief, cooling-modified PDS stage. The momentum injection after shell formation is quite limited, amounting to only $\sim 50 \%$ of $p_{\mathrm{sf}}$.

\section{NUMERICAL METHODS}

We solve the hydrodynamics equations with cooling using the Athena code, which employs an unsplit Godunov algorithm (Stone et al. 2008; Stone \& Gardiner 2009). Among the various solvers provided, we utilize the simple MUSCL-Hancock type predictor-corrector scheme (van Leer integrator; Stone \& Gardiner 2009). For robustness, we utilize piecewise linear spatial reconstruction and Roe's Riemann solver with H-correction (Sanders et al. 1998) and first order flux correction (Lemaster \& Stone 2009). We apply H-correction only for zones with maximum signal speed difference ( $\eta$-coefficient in Sanders et al. 1998) larger than $100 \mathrm{~km} \mathrm{~s}^{-1}$, to avoid artificial diffusion at the interface of the CNM and WNM where the signal speed difference is about $10 \mathrm{~km} \mathrm{~s}^{-1}$. We solve the cooling term in an operator split manner using a fully implicit method, with Newton-Raphson root finding. In addition to the Courant condition, the time step is also limited by the cooling solver, which allows only a factor of two variation in the local temperature from the value at the previous time step (Kim et al. 2008).

The governing equations are

$$
\begin{gathered}
\frac{\partial \rho}{\partial t}+\nabla \cdot(\rho \mathbf{v})=0 \\
\frac{\partial(\rho \mathbf{v})}{\partial t}+\nabla \cdot(\rho \mathbf{v} \mathbf{v}+P)=0
\end{gathered}
$$

and

$$
\frac{\partial E}{\partial t}+\nabla \cdot((E+P) \mathbf{v})=-\rho \mathcal{L}
$$


where $\rho$ is the mass density, $\mathbf{v}$ is the velocity, $E \equiv P /(\gamma-1)+\rho v^{2} / 2$ is the total energy density, $P$ is the gas pressure, and $\gamma$ is the ratio of specific heats. The gas temperature is $T=P /\left(1.1 n_{H} k_{\mathrm{B}}\right)$ for neutral gas and $T=P /\left(2.3 n_{H} k_{\mathrm{B}}\right)$ for fully ionized gas, where the hydrogen number density is $n_{H}=\rho /\left(1.4 m_{\mathrm{H}}\right)$ for $10 \%$ of Helium abundance. Since we did not follow the ionization and recombination in detail, we calculate the gas temperature using the equation for the neutral gas so that the temperature in our simulations are higher by factor of $2.3 / 1.1$ for ionized gas $\left(T \gtrsim 10^{4} \mathrm{~K}\right)$. However, the temperature in practice is only used to obtain the cooling rate, and only indirectly affects the dynamical evolution.

The net cooling rate per unit volume is $\rho \mathcal{L} \equiv n_{H}\left[n_{H} \Lambda(T)-\Gamma\right]$. For the cooling function $\Lambda(T)$ at low $\left(T<10^{4} \mathrm{~K}\right)$ and high $\left(T>10^{4} \mathrm{~K}\right)$ temperature gas, we respectively adopt fitting formulae from Koyama \& Inutsuka (2002, see also Kim et al. 2008) and piecewise power-law fits to the cooling function of Sutherland \& Dopita (1993) with collisional ionization equilibrium at solar metallicity. Heating is only applied at $T<10^{4} \mathrm{~K}$, to model photoelectric heating of the warm/cold ISM. As the photoelectric heating rate is proportional to the star formation rate, and the pressure and star formation rate are approximately proportional to each other for self-consistent solutions (Ostriker et al. 2010; Kim et al. 2011, 2013), we adopt a heating rate that varies with mean density as $\Gamma / \Gamma_{0}=\left(n_{H} / 2 \mathrm{~cm}^{-3}\right)$, where $\Gamma_{0}=2 \times 10^{-26} \mathrm{erg} \mathrm{s}^{-1}$. We neglect thermal conduction in this study (see discussion in Section 7).

We have run sets of simulations of three different types: (1) a single SN in a uniform background medium (SU), (2) a single SN in a two-phase medium (S2P), and (3) multiple SNe in a two-phase medium (M2P). For all three types of simulation, we vary the mean density of the background medium $n_{0} \equiv n_{H} / 1 \mathrm{~cm}^{-3}$ from 0.1 to 100 (10 for M2P). For our standard models, we fix the total energy of a single SN to be $E_{\mathrm{SN}}=10^{51} \mathrm{erg}$. Only for comparison to M2P models, we also have run a single-explosion model with $E_{\mathrm{SN}}=10^{52} \mathrm{erg}(\mathrm{E} 2 \mathrm{P})$. Since our main interest is on the momentum injection to the ISM at later times, we ignore the ejecta and hence the free expansion stage. We initialize the SNR in all cases within a sphere of radius $r_{\text {init }}\left(r_{\text {init }}\right.$ varies; see below). The SNR initialization region consists of all cells whose centers are at a distance $<r_{\text {init }}$ from the site of SN explosion; within the SNR initialization region, we either inject thermal energy at uniform density (most models), or apply a radially-dependent ST solution.

In order to quantify the evolution, we require definitions for the different gas components. We define the hot gas as all zones with $T>2 \times 10^{4} \mathrm{~K}$, the shell gas as zones with $T<2 \times 10^{4} \mathrm{~K}$ and $v_{r}>1 \mathrm{~km} \mathrm{~s}^{-1}$, and the ambient medium as the remainder (note that ambient gas is initially stationary for these models). For the ambient medium, we define the cold and warm neutral medium (CNM and WNM) with temperature cuts of $T<184 \mathrm{~K}$ and $T>5050 \mathrm{~K}$, respectively. Hereafter, "SNR" refers to both hot and shell gas. We define the radius of the SNR using the mass-weighted mean radius of the shell gas as $r_{\text {snr }} \equiv \sum_{\text {shell }} \rho r d V / \sum_{\text {shell }} \rho d V$ where $d V=\Delta^{3}$. This is approximately equal to the shock radius before shell formation, and slightly smaller (due to non-negligible thickness of the shell) than the shock radius after shell formation (see Figure 2). The kinetic and thermal energies are calculated as $E_{\text {kin }} \equiv \sum(1 / 2) \rho v^{2} d V$ and $E_{\text {th }} \equiv \sum[P /(\gamma-1)] d V$ for 
the SNR (hot and shell gas), and the total energy $E_{\text {tot }} \equiv E_{\text {kin }}+E_{\text {th }}$. The total radial momentum of the SNR is calculated by $p_{\mathrm{snr}} \equiv \sum \rho \mathbf{v} \cdot \hat{\mathbf{r}} d V$. We also measure the total mass of the shell and hot gas, $M_{\text {sh }} \equiv \sum_{\text {shell }} \rho d V$ and $M_{\text {hot }} \equiv \sum_{\text {hot }} \rho d V$, respectively, as well as the mean pressure of the hot gas $P_{\text {hot }} \equiv \sum_{\text {hot }} P d V / \sum_{\text {hot }} d V$.

\section{Single $\mathrm{SN}$ in Uniform Medium}

We first perform a set of numerical experiments with a single SN in a uniform, unmagnetized medium (SU models) $2^{2}$ For reference, we list in Table 1 the theoretical estimates of the shell formation time $t_{\mathrm{sf}}$ as well as the theoretical SNR radius, postshock temperature, swept-up mass, and total radial momentum at $t_{\mathrm{sf}}$ (see Equations (7), (8), (10), (11), and (17)), for each choice of ambient density in the range $n_{0}=0.1$ to 100. As we shall describe in Section 4.2, in addition to varying the physical density of the ambient medium, for our SU models we also cover a wide range of parameter space for the initial radius of the SNR $r_{\text {init }}$ and the grid spacing $\Delta$. This latter set of tests enables us to evaluate the dependence of the momentum injection on numerical resolution, and therefore establish minimum resolution requirements for modeling SN feedback in complex numerical simulations. Table 1 also lists (in parentheses) the numerically-measured values $\left(t_{\mathrm{sf}}^{\mathrm{n}}, r_{\mathrm{sf}}^{\mathrm{n}}\right.$, $M_{\mathrm{sf}}^{\mathrm{n}}$, and $p_{\mathrm{sf}}^{\mathrm{n}}$ ) obtained from high-resolution simulations, as described in Section 4.1 .

\subsection{High Resolution Reference Runs}

In this subsection, we delineate the time evolution of the SNR based on high resolution simulations of a single SN in a uniform ambient medium (SU models). We first present results of SU-n1 with grid resolution $\Delta=1 / 4 \mathrm{pc}$. The initial conditions are realized by uniformly applying the SN's thermal energy $\left(10^{51} \mathrm{erg}\right)$ within an initial radius of $r_{\text {init }}=5 \mathrm{pc}$. Figure 1 plots time evolution of (a) radius $r_{\mathrm{snr}}$, (b) deceleration parameter $\eta \equiv v_{\mathrm{snr}} t / r_{\mathrm{snr}}$, where $v_{\mathrm{snr}}$ is calculated by finite differences of the measured $r_{\mathrm{snr}}$, and $\eta$ is smoothed, (c) total $E_{\mathrm{tot}}$, thermal $E_{\mathrm{th}}$, and kinetic $E_{\mathrm{kin}}$ energies, (d) mass of hot gas $M_{\text {hot }}$ and shell gas $M_{\mathrm{sh}}$, (e) total radial momentum $p_{\mathrm{snr}}$, and (f) pressure of hot gas $P_{\text {hot }}$. The dotted and dashed lines indicate theoretical predictions of each quantity for ST and idealized PDS solutions as described in Section 2, while the vertical dot-dashed line denotes the predicted shell formation time from Equation (7). We also present in Figures 2 and 3 the radial profiles and snapshots in the $\hat{\mathbf{x}}-\hat{\mathbf{y}}$ plane at $z=0$ (XY-plane) for selected times. In Figure 2, blue dashed lines show the ST self-similar solutions at $t=0.03 \mathrm{Myr}$, while vertical dotted lines are $r_{\mathrm{snr}}$ at each epoch.

\footnotetext{
${ }^{2}$ We have also performed simulations with a magnetized medium. We find quite similar evolution and values of physical quantities overall; small differences are found only for the case of strong magnetic fields $(\beta=0.1)$ at late times. See Appendix for details.
} 

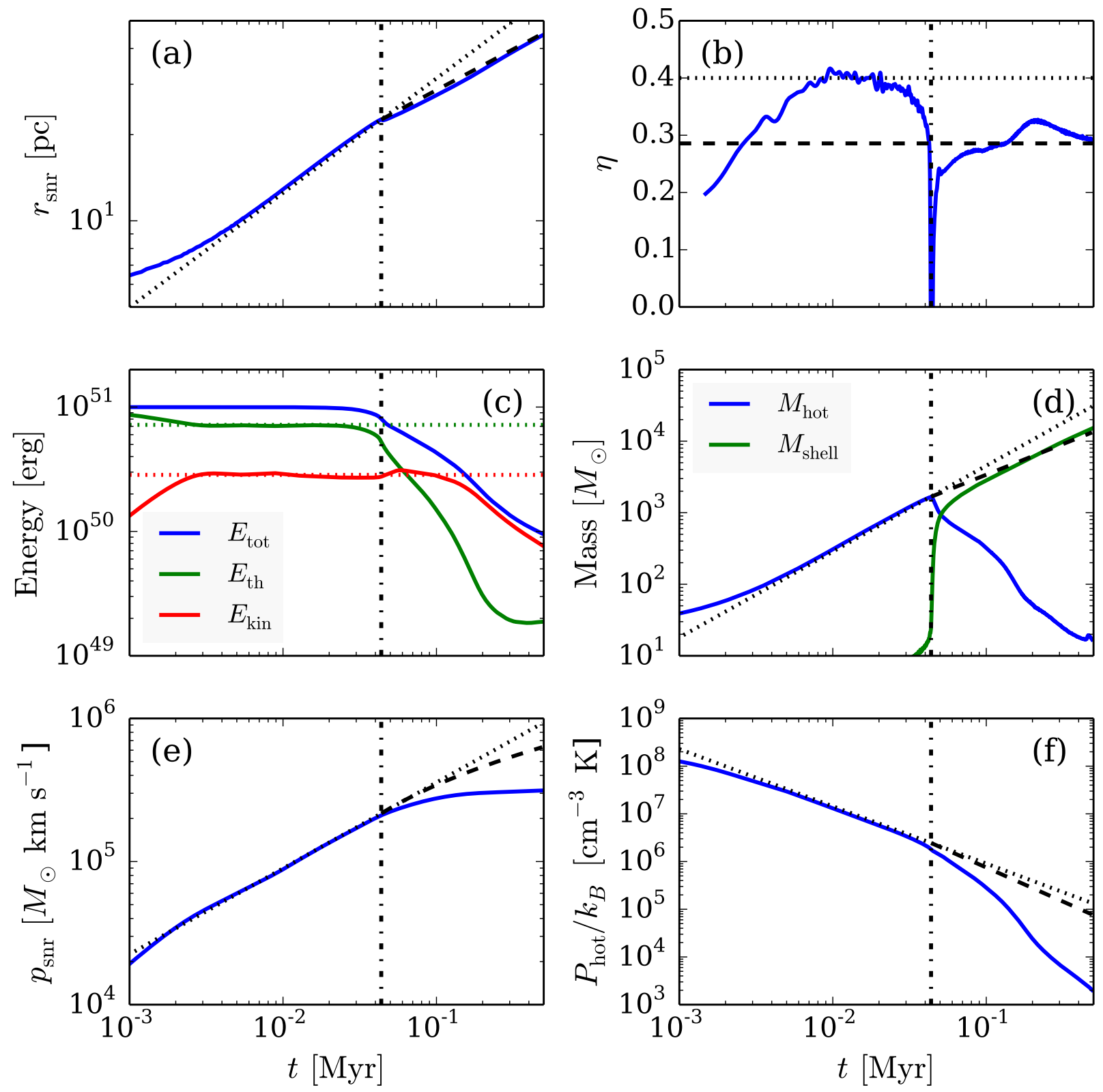

Fig. 1. - Time evolution of model SU-n1 $\left(n_{0}=1 \mathrm{~cm}^{-3}\right.$ and $\left.E_{\mathrm{SN}}=10^{51} \mathrm{erg}\right)$ : (a) mass-weighted radius, (b) deceleration parameter $\eta \equiv v_{\mathrm{snr}} t / r_{\mathrm{snr}}$, (c) total, thermal and kinetic energies, (d) mass of interior hot gas and shell, (e) total radial momentum, and (f) pressure of interior hot gas. Grid resolution is $\Delta=1 / 4 \mathrm{pc}$ and the initial SNR radius is $r_{\text {init }}=5 \mathrm{pc}$. The vertical dot-dashed lines in each panel denote the predicted shell formation time $t_{\mathrm{sf}}=4.4 \times 10^{4} \mathrm{yr}$ (Equation (7)) for this model. The dotted and dashed lines are ST and idealized PDS solutions (at $t>t_{\mathrm{sf}}$ ) for each physical quantity (see Section 2). 

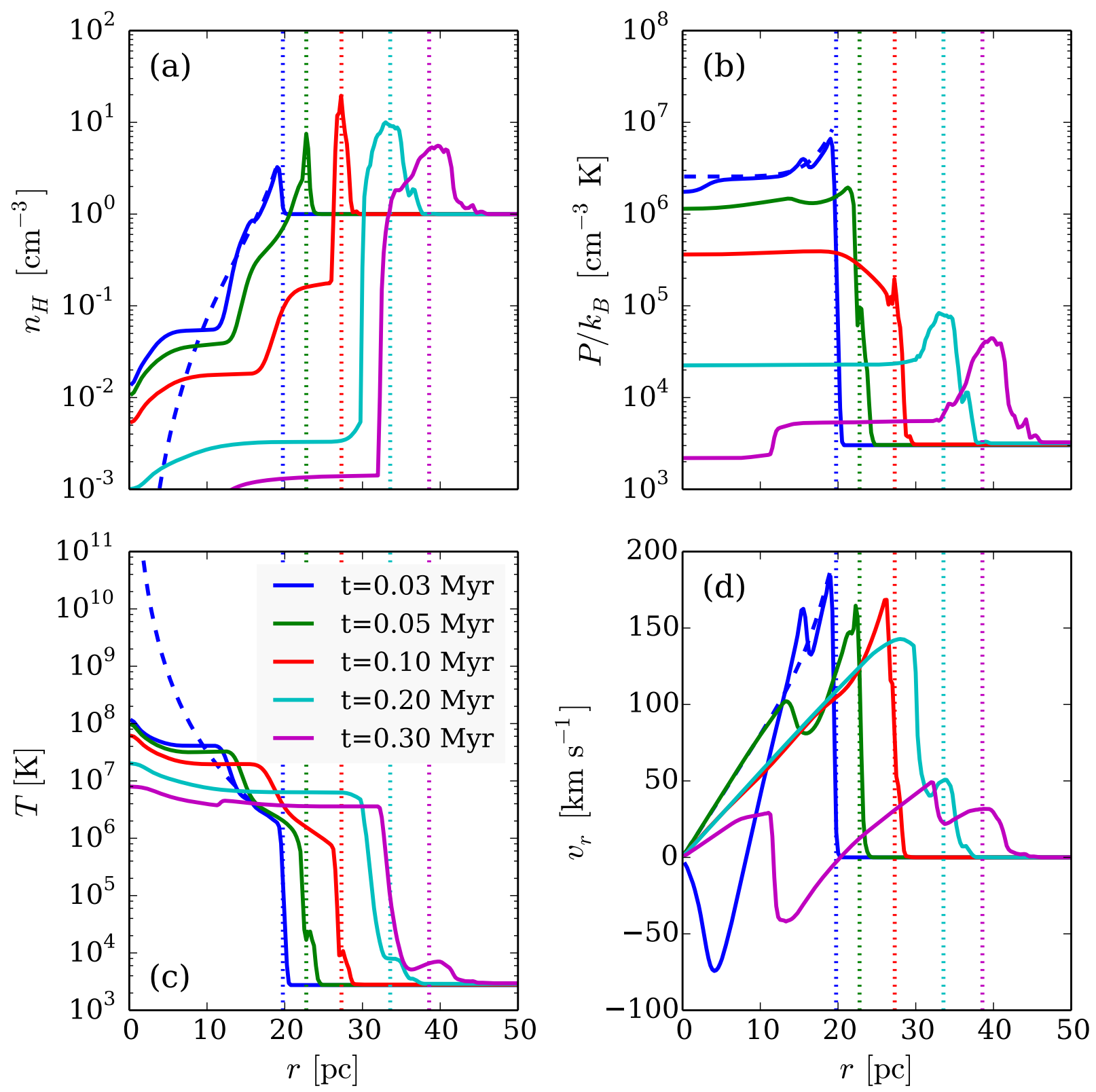

Fig. 2.- Radial profiles of (a) number density, (b) pressure, (c) temperature, and (d) radial velocity in model SU-n1 with $\Delta=1 / 4 \mathrm{pc}$ and $r_{\text {init }}=5 \mathrm{pc}$. The blue dashed line denotes the ST solution at $t=0.03$ Myr. Vertical dotted lines are the mass-weighted radius of SNR $\left(r_{\mathrm{snr}}\right)$ at each epoch, which agrees well with the shock position and mean radius of shell before and after shell formation (at $t_{\mathrm{sf}}=0.04 \mathrm{Myr}$ for this model), respectively. 


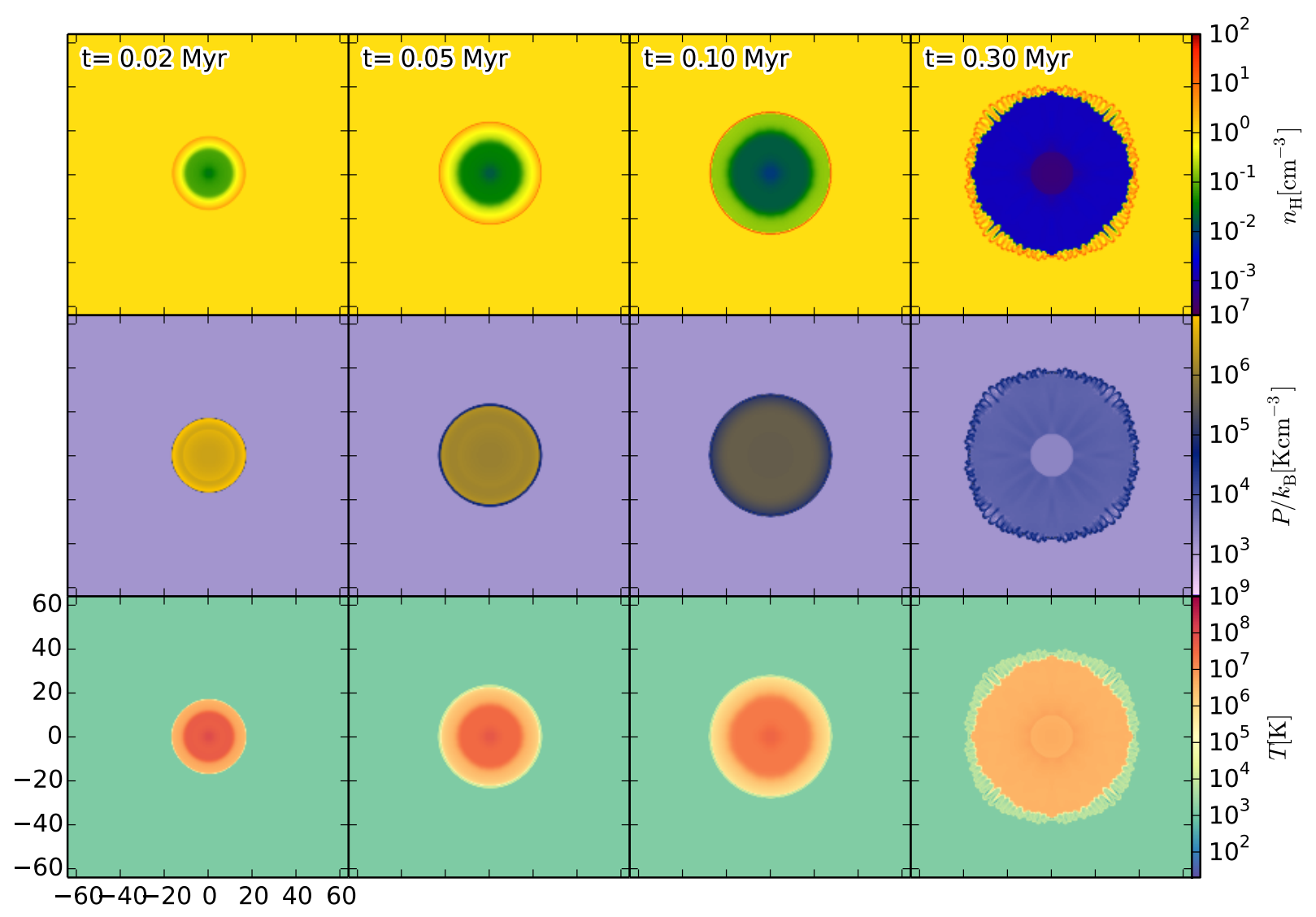

Fig. 3. - XY-slices from model SU-n1 with $\Delta=1 / 4$ pc and $r_{\text {init }}=5$ pc. From top to bottom, $\log$ arithmic color scales show number density, pressure, and temperature. From left to right, columns correspond to snapshots at $t / t_{\mathrm{sf}} \sim 1 / 2,1,2$, and 7 . A thin shell bounding the SNR is evident in the two middle columns; in the right column it has become corrugated due to dynamical instabilities. 

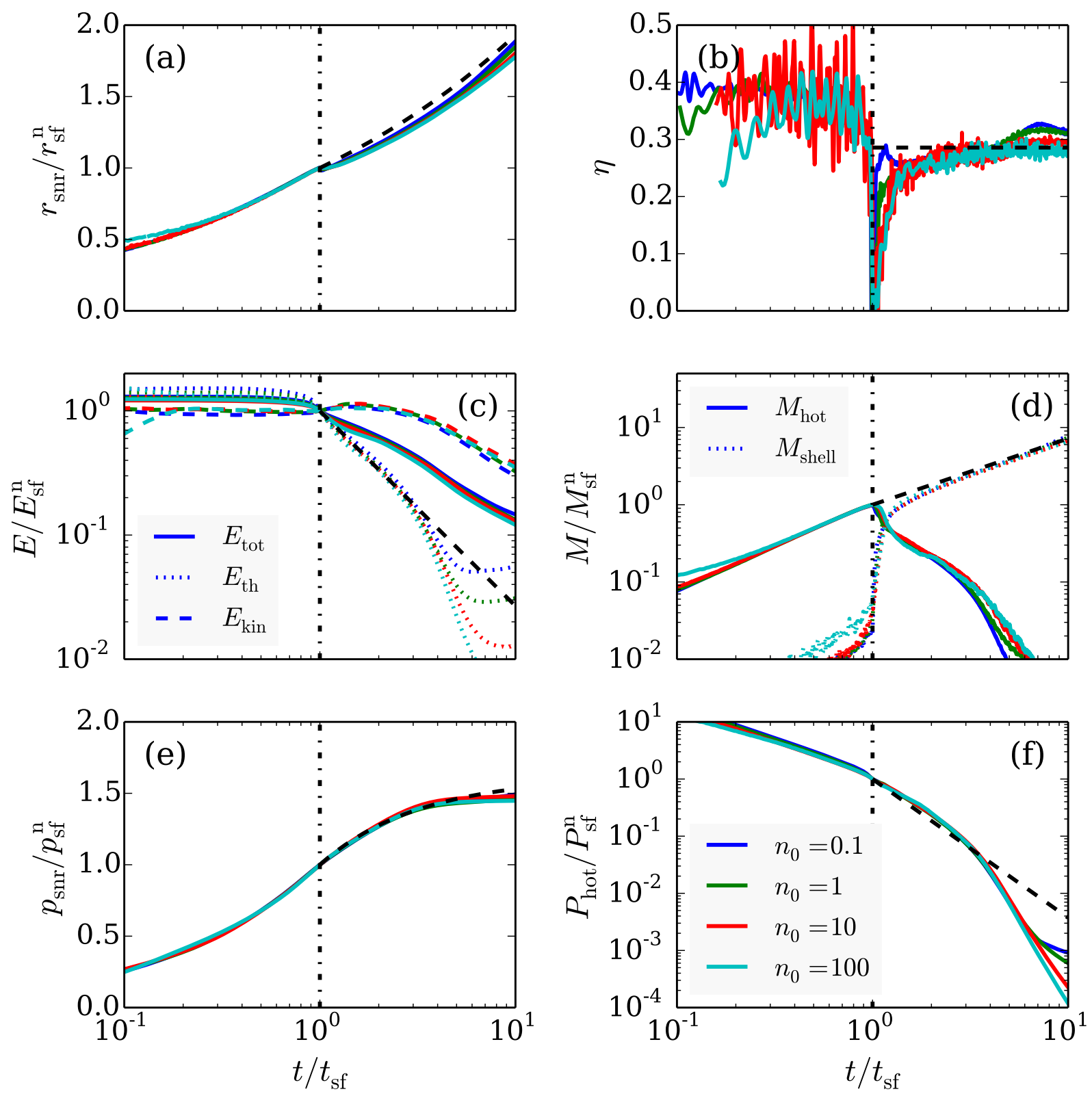

Fig. 4.- Time evolution comparison for all of the SU high-resolution models, with ambient medium density $n_{0}=0.1$ to $n_{0}=100$. Panels show (a) mass-weighted radius, (b) deceleration parameter $\eta \equiv v_{\mathrm{snr}} t / r_{\mathrm{snr}}$, (c) total, thermal and kinetic energies, (d) mass of hot gas and mass of the shell, (e) total radial momentum, and (f) pressure of interior hot gas. All physical quantities are normalized by the corresponding numerical measures at $t=t_{\mathrm{sf}}^{\mathrm{n}}$ (see text). The black dashed curves are cooling-modified PDS solutions after shell formation (see text for details). 
At $t<t_{\mathrm{sf}}$, the dynamical evolution of the SNR is characterized by the ST solutions very well. The initial SNR in the simulation has a finite size and zero kinetic energy, in contrast to an initial point source and a ratio $E_{\text {kin }} / E_{\text {th }}=0.39$ of the ST solution. However, as the shock expands into the ambient medium, radial motions are rapidly generated. After a transient of only $t \sim 2$ kyr, all the physical quantities follow the ST solution. Although the radial profiles shown in Figure 2 differ from the ST solutions at small radii $r<10 \mathrm{pc}$ due to the finite initial size, they are quite close near the shock where the most of mass and momentum are concentrated. As a consequence, there is excellent agreement with the ST solutions for all integrated physical properties, as seen in Figure 1.

As the shocked hot gas cools, a thin and dense shell is formed (see green and red lines of Figure 2 and the 2nd and 3rd columns of Figure 3. Shell formation is clearly distinguished by sudden changes of several physical quantities. At shell formation, the expansion stalls (sudden drop of $v_{\text {snr }}$ and hence $\eta$ in Figure 1(b)), the thermal energy and the mass of the hot gas begin to decrease (Figure 1(c) and (d)), and the mass of the shell gas sharply increases (Figure 1(d)). We define the numerical shell formation time, $t_{\mathrm{sf}}^{\mathrm{n}}$, as the time when $M_{\text {hot }}$ attains its maximum ${ }^{3}$. Note that the theoretical prediction of $t_{\mathrm{sf}}$ from Equation (7) agrees with the maximum of $M_{\text {hot }}$ fairly well (see vertical line in Figure 1(d) and Table 1). Since the cooling function we use is different from the single power-law cooling function used to derive Equation (7), $t_{\mathrm{sf}}^{\mathrm{n}}$ is slightly different from $t_{\mathrm{sf}}$, with slightly different dependence on $E_{\mathrm{SN}}$ and $n_{0}$ as well.

After shell formation, the pressure of hot interior gas decreases much faster than for the idealized adiabatic PDS solution, $P_{\text {hot }} \propto t^{-10 / 7}$ (dashed line in Figure 1(f)). This difference was reported by Cioffi et al. (1988), who found an additional $t^{-4 / 9}$ dependence in thermal energy and hence the pressure of the hot gas (after shell formation). Although the cooling time of the hot gas at small radii is still quite long because of its low density, the density immediately inside the shell is higher and this gas is able to cool. In addition, the velocity interior to the shell exceeds that of the shell itself (see red, cyan, and magenta lines in Figure 2(d)), so that interior hot gas continues to accumulate, condense, and cool at the inner surface (back) of the shell. With a steadily decreasing mass of hot gas, the interior pressure is ever lower than that of the idealized PDS solution (which assumes a constant interior mass), adding much less momentum (compare dashed and solid lines in Figure 1(e)). The evolution of radius with time nevertheless seems to be roughly consistent with the PDS exponent $\eta=2 / 7$ (or 3/10 for the offset power-law fit in Cioffi et al. 1988; see Figures 1(a) and (b)), but this can also be explained as a transition from the ST to MCS phase, i.e. from $\eta=0.4$ to $\eta=0.25$ (Blondin et al. 1998; Bandiera \& Petruk 2004). Since the hot gas mass drops very rapidly, the duration of the "classical" PDS stage is negligible.

As most of the hot gas has already piled into the shell by $t>(2-3) t_{\mathrm{sf}}$, the hot gas pressure is no longer high enough to drive the shell outward and add appreciable momentum (cyan and magenta

\footnotetext{
3 Thornton et al. (1998) use the time of maximum luminosity, $t_{0}$, to identify shell formation; our definition based on the hot gas mass is in practice quite similar.
} 
lines in Figure 2(b); Figure 1(e)). In addition, the shell becomes thicker at this stage so that the thin shell approximation is no longer valid. The shell is broken up in this high resolution simulation (fourth column of Figure 3) due to the pressure-driven thin shell overstability and/or nonlinear thinshell instability (Vishniac 1983, 1994, Blondin et al. 1998). I4 Although we do not explicitly assign perturbations to our models, at the initial interface between the SNR and ISM there are unavoidably grid-scale nonlinear perturbations due to the mapping of a sphere to Cartesian coordinates. This seeds instability, which develops into strong corrugations. Note that this instability is less prominent for realistic cases, in which the multiphase structure of the ambient ISM dominates in shaping the shell (see Section 6).

In models SU-n0.1, SU-n10, and SU-n100, we conduct analogous simulations to model SU-n1, covering the range of ambient density of $n_{0}=0.1$ to 100 . For the high-resolution models with $n_{0}=0.1,1,10$, and 100 , we set $\Delta=1,1 / 2,1 / 4$, and $1 / 8 \mathrm{pc}$, respectively, and $r_{\text {init }}=4 \Delta$; these choices are based on our convergence study (see Section 4.2 ).

Figure 4 plots the same quantities as in Figure 1, but normalized using the corresponding numerical measures at $t_{\mathrm{sf}}^{\mathrm{n}}$. As before, this $t_{\mathrm{sf}}^{\mathrm{n}}$ is defined by the time when the hot gas mass attains its maximum value. Note that $t_{\mathrm{sf}}^{\mathrm{n}}, r_{\mathrm{sf}}^{\mathrm{n}}, M_{\mathrm{sf}}^{\mathrm{n}}$, and $p_{\mathrm{sf}}^{\mathrm{n}}$ given in Table 1 for these high-resolution models can be fitted by, respectively,

$$
\begin{gathered}
t_{\mathrm{sf}}^{\mathrm{n}}=4.0 \times 10^{4} \text { yr } n_{0}^{-0.59}, \\
r_{\mathrm{sf}}^{\mathrm{n}}=22.1 \text { pc } n_{0}^{-0.43}, \\
M_{\mathrm{sf}}^{\mathrm{n}}=1550 M_{\odot} n_{0}^{-0.29},
\end{gathered}
$$

and

$$
p_{\mathrm{sf}}^{\mathrm{n}}=2.00 \times 10^{5} M_{\odot} \mathrm{km} \mathrm{s}^{-1} n_{0}^{-0.15}
$$

Figure 4 demonstrates that the overall (normalized) evolution is remarkably similar, irrespective of the ambient density. Since the normalization factors are close to the analytic estimates from the ST solution and the early evolution is expected to converge to the ST solution, this agreement is trivial at $t<t_{\mathrm{sf}}^{\mathrm{n}}$.

However, even though the classical PDS solution is not realized in the numerical models, the characteristic behavior after shell formation is nevertheless essentially the same over a wide range of densities. Model evolution is thus "congruent" for varying ambient density when rescaled relative to the SNR properties at $t_{\mathrm{sf}}^{\mathrm{n}}$.

\footnotetext{
${ }^{4}$ The instability of radiative shells was carefully studied by Blondin et al. (1998). Immediately after shell formation, a radiative reverse shock emerges, resulting in a thin shell that is bounded by shocks on both sides. This is susceptible to the nonlinear thin shell instability (Vishniac 1994). For the low density case, similar to our fiducial model SU-n1, however, Blondin et al. (1998) showed that the reverse shock soon becomes non-radiative and leaves the shell. In this situation, the shell is bounded by a forward shock and interior pressure, which can lead to the pressure-driven thin shell overstability (Vishniac 1983).
} 
In order to describe the transition between ST and PDS phases smoothly, Cioffi et al. (1988) introduced an analytic description using an offset power-law for radius and a complex fitting model for the thermal energy with additional time dependence. Since energy loss by radiative cooling begins somewhat earlier than the shell formation, they also arbitrarily defined a PDS time $t_{\text {PDS }} \equiv$ $t_{\mathrm{sf}} / e$. Here, instead of using the approach of Cioffi et al. (1988), we introduce a simpler analytic formula to describe the post-shell formation phase separately. As already seen in Figure 1, the evolution of radius agrees reasonably well with the classical PDS solution. We thus simply adopt $r_{\mathrm{snr}}=r_{\mathrm{sf}}\left(t / t_{\mathrm{sf}}\right)^{2 / 7}$ (other exponents between 0.4 and 0.25 give similar results). Following Cioffi et al. (1988), we describe the evolution of thermal energy after shell formation with an additional time dependence as

$$
E_{\mathrm{th}}=0.8 E_{\mathrm{th}, \mathrm{ST}}\left(\frac{r_{\mathrm{sf}}}{r_{\mathrm{snr}}}\right)^{2}\left(\frac{t_{\mathrm{sf}}}{t}\right),
$$

where $E_{\mathrm{th}, \mathrm{ST}}=0.717 E_{\mathrm{SN}}$ is the thermal energy at the ST stage, and the factor " 0.8 " arises from precooling before the shell formation (see dotted lines in Figure 4(c)).

The factor $t_{\mathrm{sf}} / t$ is chosen to give a good match to the numerical solutions for the time dependence of $E_{\mathrm{th}}$. One might expect $E_{\mathrm{th}}$ to decrease after shell formation proportional to the product of $M_{\text {hot }}$ and the interior temperature. The mass-weighted mean temperature of hot gas is nearly constant in this phase. Thus, $M_{\text {hot }}$ and $E_{\text {th }}$ show similar time dependence (approximately $\left.\propto\left(t / t_{\mathrm{sf}}\right)^{-1.64}\right)$ until the thermal energy of shell gas begins to dominate at $t>4 t_{\mathrm{sf}}$.

Using Equation (26), the effective pressure on the shell is then

$$
P_{\text {hot }}=\frac{E_{\mathrm{th}}}{2 \pi r_{\mathrm{snr}}^{3}}=0.8 P_{\mathrm{sf}}\left(\frac{r_{\mathrm{sf}}}{r_{\mathrm{snr}}}\right)^{5}\left(\frac{t_{\mathrm{sf}}}{t}\right) .
$$

We then have an expression for the momentum similar to Equation (18):

$$
p_{\mathrm{snr}}=p_{\mathrm{sf}}\left[1+0.53 \int_{1}^{t_{*}} \frac{d t_{*}}{r_{*}^{3} t_{*}}\right]=p_{\mathrm{sf}}\left[1+0.62\left(1-t_{*}^{-6 / 7}\right)\right] .
$$

Note that with this modified, non-adiabatic PDS stage, the total momentum is finite, $p_{\mathrm{snr}} \rightarrow 1.6 p_{\mathrm{sf}}$ as $t \rightarrow \infty$; only $60 \%$ of $p_{\text {sf }}$ is added during the post shell formation stages. The black dashed lines in Figure 4 represent the modified PDS solution described above. The final momenta $\left(p_{\text {final }}\right)$ measured at $t=10 t_{\mathrm{sf}}^{\mathrm{n}}$ are in fact still smaller than $1.6 p_{\mathrm{sf}}$ since no momentum is acquired after $(4-5) t_{\mathrm{sf}}^{\mathrm{n}}$ as the hot gas pressure becomes comparable to the shell gas pressure. We find that $p_{\text {final }} / p_{\mathrm{sf}}^{\mathrm{n}}=1.49,1.46$, 1.48 , and 1.45 for $n_{0}=0.1,1,10$, and 100 , respectively. In physical units, the final momentum can be fitted as

$$
p_{\text {final }}=2.95 \times 10^{5} M_{\odot} \mathrm{km} \mathrm{s}^{-1} n_{0}^{-0.16} .
$$

It is interesting to compare our numerical results with earlier studies based on one-dimensional spherical models. Blondin et al. (1998) have measured the shell formation time in terms of the "transition" time (Equation (3) of their paper; simply defined by equating the cooling time with 
the age of the SNR). For $n_{0}=0.084,0.84$, and 84, their numerical measures of the shell formation time are $1.35,1.23$, and 1.61 times our $t_{\mathrm{sf}}^{\mathrm{n}}$ for $n_{0}=0.1,1$, and 100 , respectively.

A wide parameter space of density and metallicity has been covered by Thornton et al. (1998). In their work, the numerical measures of the shell formation time ( $t_{0}$ in their notation), agree with our $t_{\mathrm{sf}}^{\mathrm{n}}$ within $20 \%$ for corresponding density. More interestingly, their ratios of total final momentum (measured at $13 t_{0}$ ) to the momentum at $t_{0}$ are 1.5-1.6 for $n_{H}=0.1$ to $100 \mathrm{~cm}^{-3}$, similar to our findings.5 Even for more extreme parameters with $n_{H}=10^{-3}$ and $10^{3} \mathrm{~cm}^{-3}$ or different metallicities, the ratios still remain around 1.7-2.

\subsection{Condition for Convergence}

As shown in the previous section, with sufficiently high resolution and a small enough initial size for the SNR, we are able to reproduce in our 3D simulations the evolution of radiative SNR as seen in previous 1D spherical models (e.g., Cioffi et al. 1988; Thornton et al. 1998; Blondin et al. 1998). Our models show a clear ST stage, but the post shell formation phase differs from the "classical" PDS solution, similar to findings from previous numerical work. Within $\sim 4 t_{\text {sf }}$, the total radial momentum of the SNR asymptotes to a value $\sim 1.5$ times the momentum at the time of shell formation. The above high-resolution SU simulations provide a benchmark, and in this subsection, we repeat the SU simulations for a wide range of numerical grid resolution $(\Delta)$ and initial size of SNR $\left(r_{\text {init }}\right)$. Our goal is to determine minimum numerical requirements needed to reproduce the main features of expanding SNRs on Cartesian grids, for the purposes of tracking SN feedback effects in large-scale ISM and galactic models.

To test dependence on grid resolution $\Delta$, we compare results for key physical quantities in model SU-n1 at $t / t_{\mathrm{sf}}=1 / 2,1,2$, and 5 . Figure 5 plots (a) total momentum, (b) kinetic energy, (c) hot gas pressure, and (d) hot gas mass, for a range $\Delta / r_{\text {sf }}=0.01$ to 1.5 . We assign $r_{\text {init }}=\Delta$ as $\Delta$ varies, such that the initial thermal energy of SN is distributed among eight zones nearest to the center of the simulation domain (the SN explosion occurs at a grid corner). All physical quantities are normalized by the values of the ST solution at $t_{\mathrm{sf}}$. Here, we use the analytic estimates of $t_{\mathrm{sf}}$ and other variables rather than numerically determined values since the numerical measures vary from model to model, whereas for a resolution study we require a fixed reference.

For $\Delta / r_{\text {sf }}<1 / 10$ and $1 / 3$, both the momentum and kinetic energy are converged respectively within $20 \%$ and $30 \%$ of the highest-resolution results at every evolutionary stage. At late stages after shell formation, the hot gas mass depends on the resolution since the mass is concentrated near

\footnotetext{
${ }^{5}$ In Thornton et al. (1998), the total momentum is not explicitly provided. Using the values presented in their Tables 1-4, we calculate the total momentum as $p_{\mathrm{snr}} \equiv\left(2 M_{R} E_{R_{\mathrm{kin}}}\right)^{1 / 2}$, where $M_{R}$ and $E_{R_{\mathrm{kin}}}$ are mass and kinetic energy of the SNR, respectively. Note that this definition is consistent with direct integration of the specific momentum if most of the mass is concentrated in a narrow region, which is true for both ST and post-shell formation phases.
} 

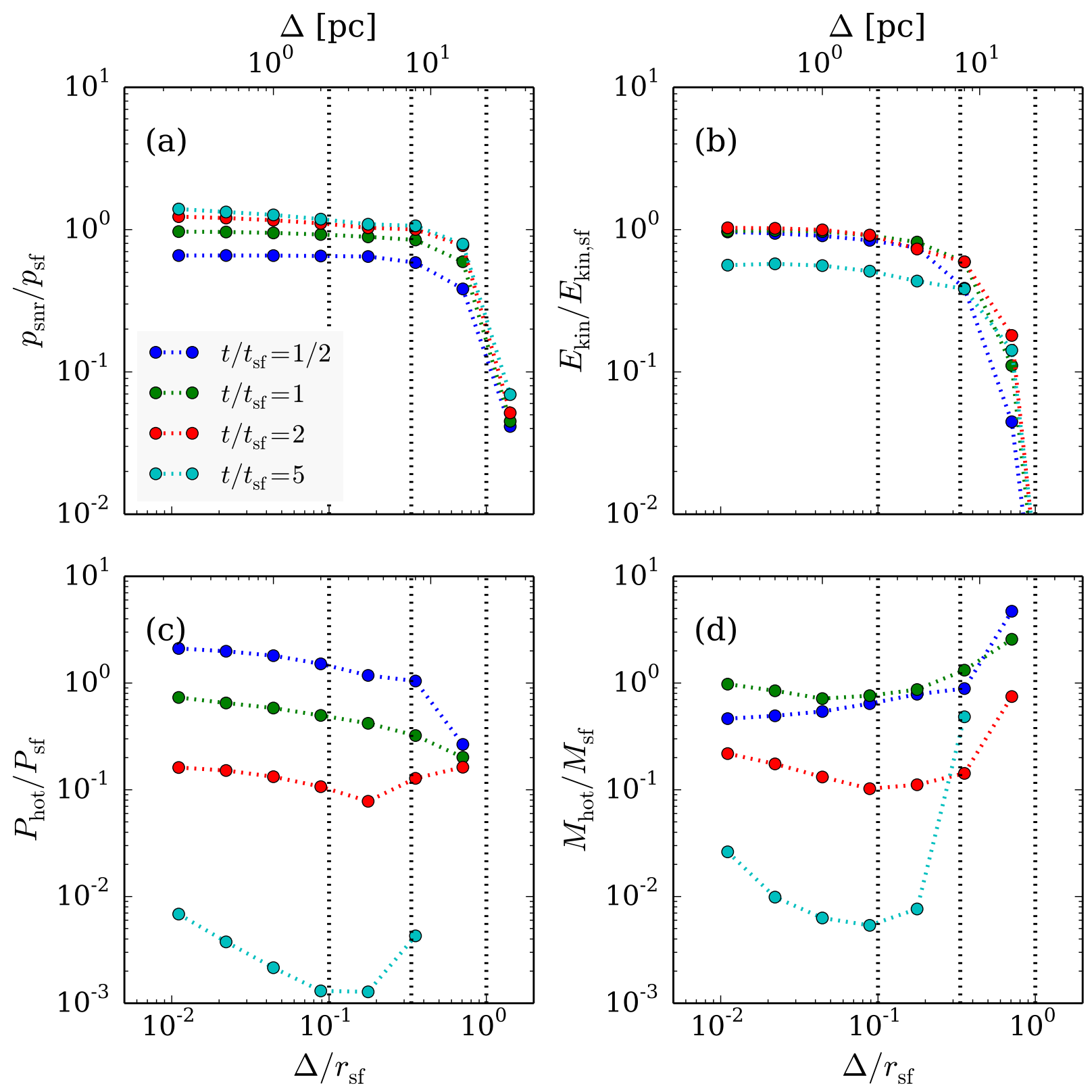

Fig. 5.- Convergence plot at varying grid resolution $\Delta$, showing (a) radial momentum, (b) kinetic energy, (c) hot gas pressure, and (d) hot gas mass, for model SU-n1. Physical quantities are measured at $t / t_{\mathrm{sf}}=1 / 2$ (blue), 1 (green), 2 (red), and 5 (cyan). All quantities are normalized based on the ST solution at $t_{\mathrm{sf}}$. Top and bottom axes show grid scale in pc and relative to $r_{\mathrm{sf}}$. For reference, vertical dotted lines denote $\Delta / r_{\text {sf }}=1 / 10,1 / 3$, and 1 . Evidently, $\Delta / r_{\text {sf }}=1 / 10$ and $1 / 3$ are practical criteria for robust and crude convergence, respectively. 

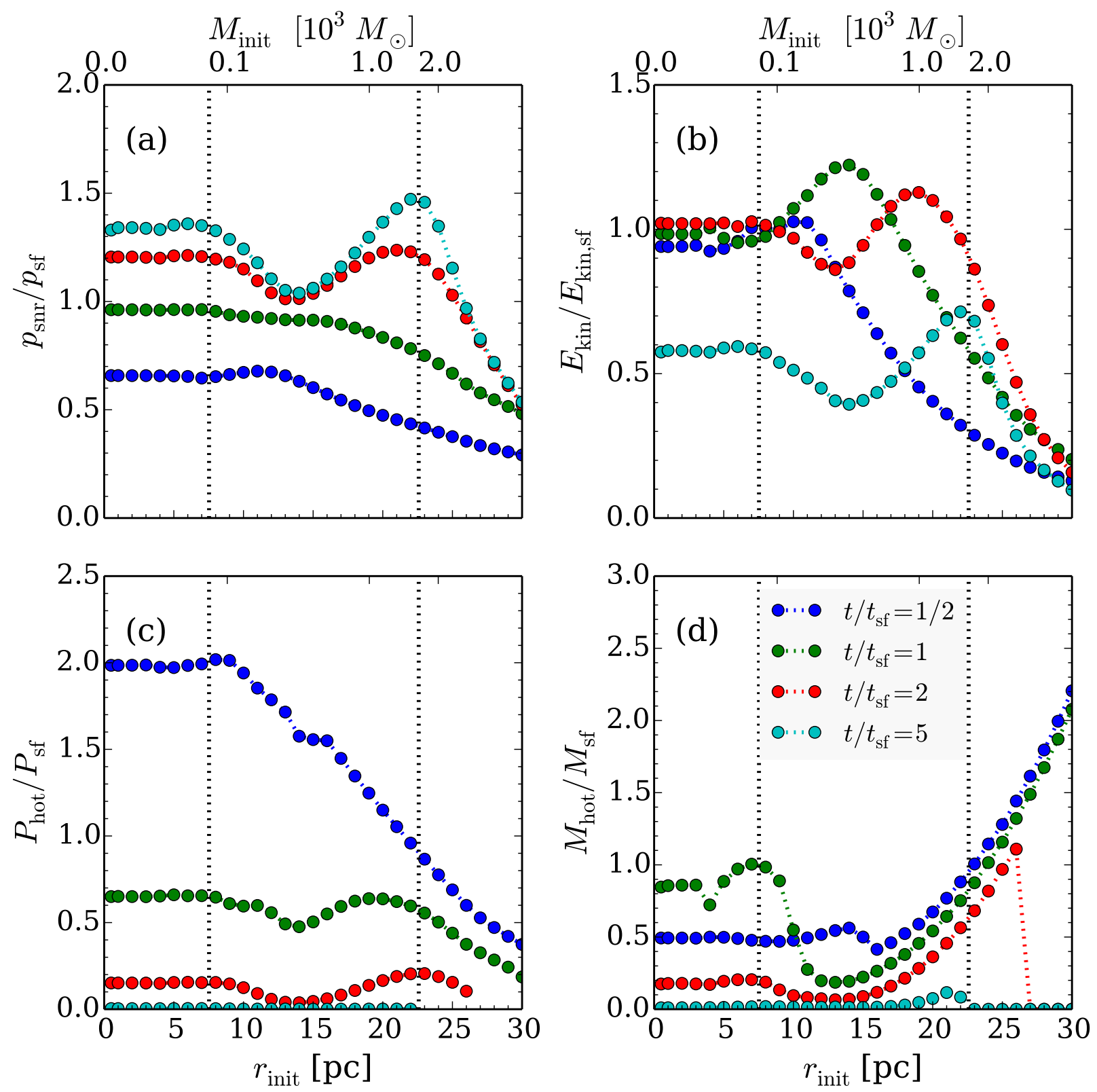

Fig. 6. - Convergence plot for varying initial SNR radius, $r_{\text {init }}$, showing (a) radial momentum, (b) kinetic energy, (c) hot gas pressure, and (d) hot gas mass, for model SU-n1. Physical quantities are measured at $t / t_{\mathrm{sf}}=1 / 2$ (blue), 1 (green), 2 (red), and 5 (cyan). All quantities are normalized based on the ST solution at $t_{\mathrm{sf}}$. Top and bottom axes show $M_{\text {init }}$ and $r_{\text {init }}$ for the SNR. For reference, vertical dotted lines denote $r_{\text {init }} / r_{\text {sf }}=1 / 3$ and 1 . All tests use $\Delta=1 / 2 \mathrm{pc} \sim 0.02 r_{\mathrm{sf}}$. For all evolutionary stages and all quantities, convergence is clear for $r_{\text {init }} / r_{\mathrm{sf}}<1 / 3$. Although the final momentum is within $\sim 30 \%$ of the correct value for all cases with $r_{\text {init }} / r_{\text {sf }}<1$, the hot gas properties are far from correct at $t<t_{\mathrm{sf}}$ for $1 / 3<r_{\text {init }} / r_{\mathrm{sf}}<1$. 

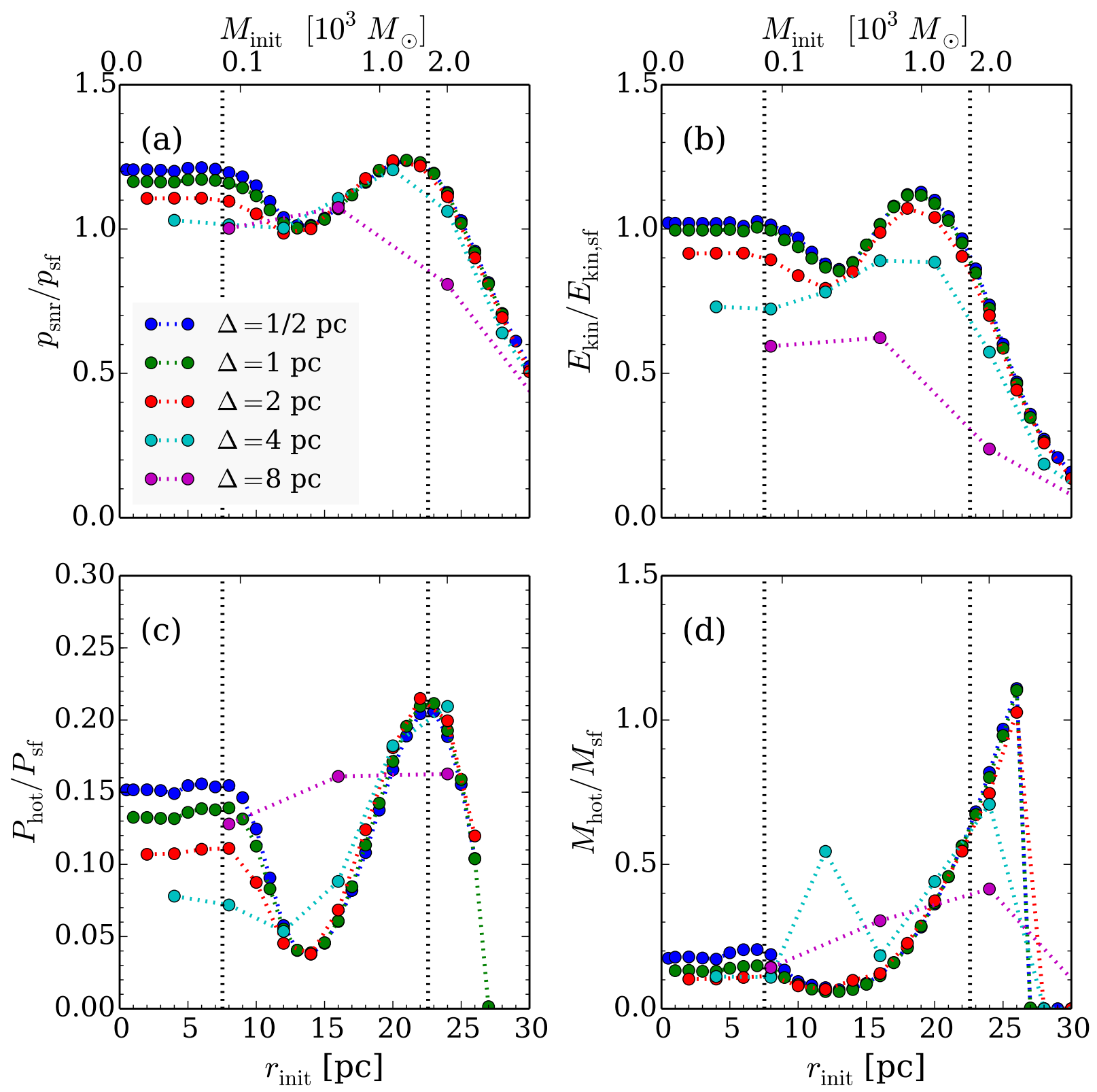

Fig. 7. - Same as Figure 6 but testing the effect of varying resolution $\Delta$ at $t / t_{\mathrm{sf}}=2$. Convergence with respect to the initial SNR size is satisfied for $r_{\text {init }} / r_{\mathrm{sf}}<1 / 3$ at all $\Delta$, but converged values fall below the high-resolution benchmarks as the resolution gets poorer. 

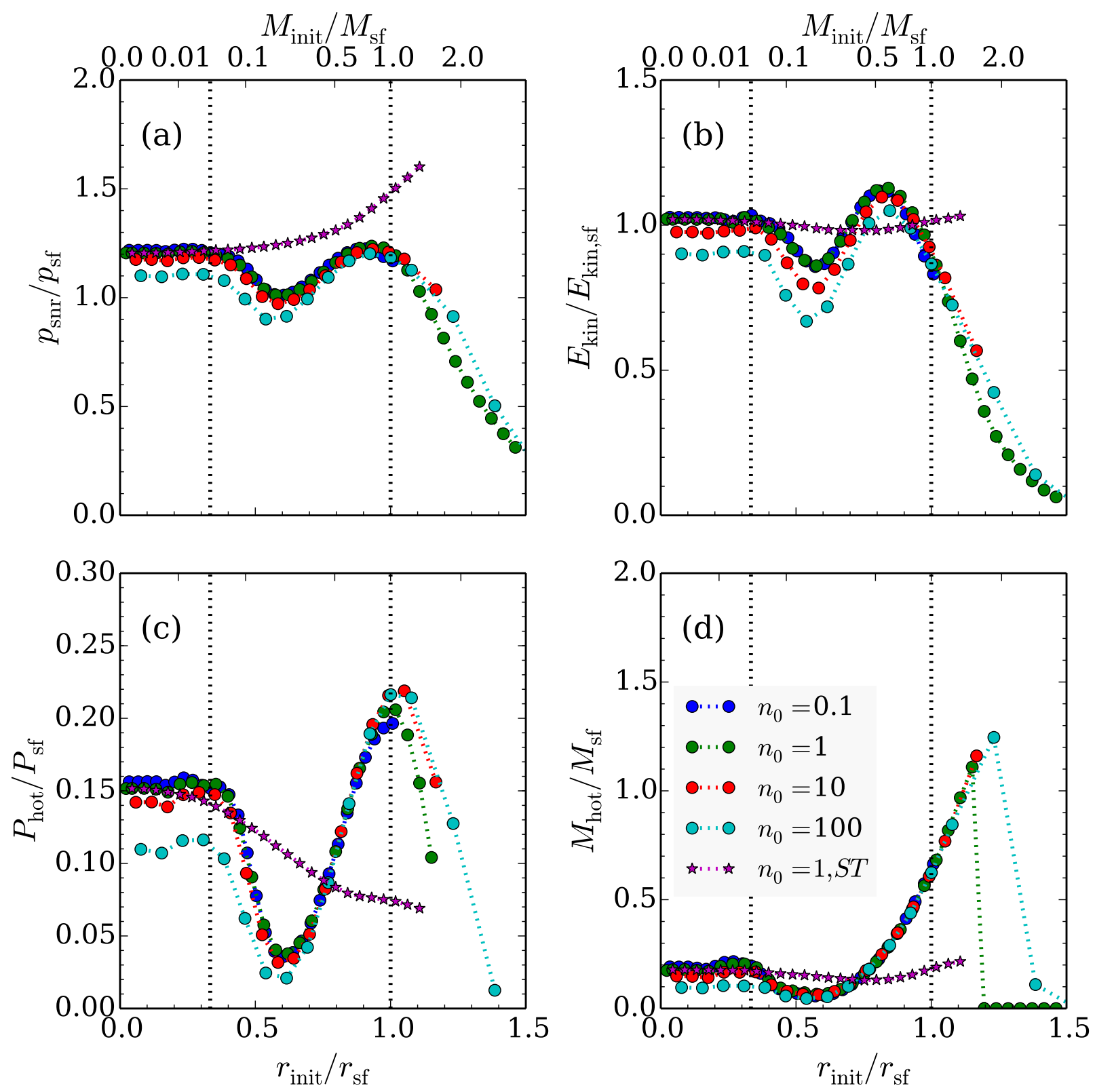

Fig. 8. - Same as Figure 6 but for different ambient density, and only showing results for $t / t_{\mathrm{sf}}=2$. The grid resolutions are $\Delta=1,1 / 2,1 / 4$, and $1 / 8 \mathrm{pc}$ for $n_{0}=0.1,1,10$, and 100, respectively, corresponding to $\Delta / r_{\mathrm{sf}}=0.015,0.02,0.03$, and 0.04 . The bottom and top x-axes are normalized by the radius and mass at the shell formation time for the corresponding density. In addition to models initiated with thermal energy only, models initialized with Sedov-Taylor profiles are shown for $n_{0}=1$ (magenta stars). Convergence requires $r_{\text {init }} / r_{\mathrm{sf}}<1 / 3$ irrespective of ambient density. 
the boundary of the hot and shell gas (and is sensitive to the exact definition of "hot" vs "shell" at later times when the hot gas mass is small), but shows very good convergence at $t / t_{\mathrm{sf}}=1$ for $\Delta / r_{\mathrm{sf}}<1 / 3$. The convergence of $P_{\text {hot }}$, which is volume weighted, is as good as $<30 \%$ and $<50 \%$ for $\Delta / r_{\mathrm{sf}}<1 / 10$ and $1 / 3$, respectively, omitting $t / t_{\mathrm{sf}}=5$ when the hot gas has negligible effect on dynamics. For $\Delta>r_{\text {sf }}$, the SNR cools down completely in a short time, so that there is no hot gas even at $t / t_{\mathrm{sf}}<1 / 2$; the momentum is $<10 \%$ and kinetic energy is $<1 \%$ of the converged values. Thus, the dynamical effect of SN feedback is impossible to model via injection of thermal energy if the grid resolution exceeds $r_{\text {sf }}$ (see also Kimm \& Cen 2014). This is the origin of the "overcooling" problem cited in many numerical simulations of galaxy formation (e.g., Katz 1992), which at best resolve scales several tens of pc and above.

In addition to grid resolution, prescriptions for SN feedback in large-scale simulations that are based on thermal energy injection must also specify an initial SNR radius, $r_{\text {init }}$. By varying $r_{\text {init }} / r_{\text {sf }}$, we also have assessed convergence with respect to this numerical parameter. For these tests, we use the SU-n1 model and adopt $\Delta=1 / 2 \mathrm{pc} \sim 0.02 r_{\mathrm{sf}}$. Figure 6 plots (a) radial momentum, (b) kinetic energy, (c) hot gas pressure, and (d) hot gas mass, at several different times, as a function of initial radius (or initial enclosed mass $M_{\text {init }}$ ). It is clear that converged results are obtained at every evolutionary stages for $r_{\text {init }} / r_{\mathrm{sf}}<1 / 3$. Although momentum and kinetic energy that are not too far from correct values can also be obtained with $r_{\text {init }} \sim r_{\text {sf }}$, the evolutionary history and internal profiles are completely different from the converged, resolved solutions if $r_{\text {init }} / r_{\mathrm{sf}}>1 / 3$.

We have confirmed that the convergence criterion $r_{\text {init }} / r_{\text {sf }}<1 / 3$ is valid for different resolutions and ambient density. Figures 7 and 8 plot the same quantities as in Figure 6 at $t / t_{\mathrm{sf}}=2$, for different resolutions $\Delta$ and ambient density. Similar convergence trends are shown for lower resolution simulations and for a wide range of varying ambient density. With poorer resolution, all quantities converged to smaller values than in the high-resolution benchmark models, as seen in Figure 5. If, instead of initializing with pure thermal energy inside $r_{\text {init }}$, the initial conditions instead employ the ST profile, then the final momentum is converged out to $r_{\text {init }} / r_{\mathrm{sf}} \sim 1 / 2$. However, initializing with the ST profile results in momentum exceeding the benchmark value if $r_{\text {init }}$ is comparable to or greater than $r_{\mathrm{sf}}$.

We conclude that in order to obtain both convergence and accuracy (consistent convergence), SNR must be initialized with both small enough radius $r_{\text {init }} / r_{\text {sf }}<1 / 3$, and high enough grid resolution. The converged values remain within roughly $25 \%$ and $50 \%$ of benchmarks for all physical quantities with $\Delta / r_{\mathrm{sf}}<1 / 10$ and $1 / 3$, respectively. In terms of injected momentum, $\Delta / r_{\mathrm{sf}}<1 / 10$ and $1 / 3$ respectively give converged values within $5 \%$ and $13 \%$ of benchmarks at $t / t_{\mathrm{sf}}=1$, and $18 \%$ and $25 \%$ of benchmarks at $t / t_{\mathrm{sf}}=10$. The final momentum is marginally satisfactory when $r_{\text {init }} \sim r_{\text {sf }}$ and $\Delta / r_{\text {sf }} \sim 1 / 3$ are adopted. Any value $r_{\text {init }}>r_{\text {sf }}$, initialized with either pure thermal energy or a ST profile, gives poor results. 


\section{Single SN in Two-Phase Cloudy Medium}

In the real ISM, the ambient gas around SNe is not uniform, but clumpy. The immediate environment will be a photoionized H II region that is also impacted by the progenitor's stellar winds. At larger scales, comparable to $r_{\mathrm{sf}}$, SNe may propagate either into dense molecular gas of a parent cloud (if it has not yet been dispersed), into the cloudy warm/cold atomic medium, or into very low density hot gas produced by previous generations of SNRs. The case of propagation into the warm/cold atomic ISM is especially important to quantify, as SNRs are believed to be the main source of turbulence in this phase of the ISM.

The atomic ISM $\left(T<10^{4} \mathrm{~K}\right)$ is regulated by two dominant cooling processes, atomic fine structure line cooling and Ly $\alpha$, yielding a two-phase medium with temperature near $10^{2} \mathrm{~K}$ and $10^{4}$, respectively; grain-photoelectric net heating dominates nearly irrespective of the gas temperature (Wolfire et al. 1995b, 2003). For a range of pressures, there is a third possible equilibrium temperature in between the warm and cold phases, but this has relatively low occupation due to the thermal instability, with a growth time $\sim$ Myr that is shorter than large-scale dynamical times in the ISM (Field 1965; Field et al. 1969; Wolfire et al. 1995b; Piontek \& Ostriker 2004, Kim et al. 2008). This results in an ISM structure consisting of cold, dense clouds embedded in a background of warm, rarefied gas. The warm and cold medium densities differ by two orders of magnitude.

To study propagation of SNRs into atomic ISM gas, we first construct two-phase "background states" via nonlinear simulations of the thermal instability (TI runs). The initial conditions for each TI run is a thermally unstable equilibrium with $10 \%$ density perturbations. For each different mean density $\left(n_{0}=0.1-100\right), \Gamma \propto n_{0}$ (as in the SU models; this scaling is consistent with selfregulated star formation), which makes the initial state thermally unstable ${ }^{6}$ We follow each TI run long enough to obtain a saturated two-phase state with pressure balance. We then explode a $\mathrm{SN}$ at the center of the simulation domain.

As the details of the cloudy structure that a SNR encounters affects its propagation, for each mean density in the S2P model set we use 10 different realizations of the cloudy background state (from TI runs with different perturbation seeds). Table 2 lists from 3rd to 5 th columns the mean densities $\left(\bar{n}_{H}\right)$, volume fractions $\left(f_{V}\right)$, and mass fractions $\left(f_{M}\right)$ of CNM $(T<184 \mathrm{~K})$ and WNM $(T>5050 \mathrm{~K})$ gas in the saturated state, averaged over these 10 realizations. The temperature cuts are determined by the maximum and minimum temperatures of the stable CNM and WNM, respectively, for our choice of cooling function (see Koyama \& Inutsuka 2002; Kim et al. 2008). Variations of the mean values in different realizations are less than $1 \%$. Note that the sum of $f_{V}$ and $f_{M}$ is not 1 since a small fraction of gas resides in the cloud interface. As $\Gamma \propto n_{0}$, the volume

\footnotetext{
${ }^{6}$ Conditions at the highest density $n_{0}=100 \mathrm{~cm}^{-3}$ are more likely to be found in the molecular ISM than the atomic ISM. However, as the chemical state of the ambient gas is unimportant when shocks are strong, it is useful to consider this case as representative of the high-density, clumpy conditions that are present in the centers of normal disk galaxies and more pervasively in starbursts.
} 
Table 1. Physical Quantities at Shell Formation for Uniform Ambient Density

\begin{tabular}{lcccccc}
\hline \hline Model & $\begin{array}{c}n_{H} \\
{\left[\mathrm{~cm}^{-3}\right]}\end{array}$ & $\begin{array}{c}t_{\mathrm{sf}}\left(t_{\mathrm{sf}}^{\mathrm{n}}\right) \\
{[\mathrm{kyr}]}\end{array}$ & $\begin{array}{c}r_{\mathrm{sf}}\left(r_{\mathrm{sf}}^{\mathrm{n}}\right) \\
{[\mathrm{pc}]}\end{array}$ & $\begin{array}{c}T_{\mathrm{sf}} \\
{\left[10^{6} \mathrm{~K}\right]}\end{array}$ & $\begin{array}{c}M_{\mathrm{sf}}\left(M_{\mathrm{sf}}^{\mathrm{n}}\right) \\
{\left[10^{3} M_{\odot}\right]}\end{array}$ & $\begin{array}{c}p_{\mathrm{sf}}\left(p_{\mathrm{sf}}^{\mathrm{n}}\right) \\
{\left[10^{5} M_{\odot} \mathrm{km} \mathrm{s}^{-1}\right]}\end{array}$ \\
\hline SU-n0.1 & 0.1 & $156(150)$ & $59.5(58.5)$ & 0.31 & $3.08(2.96)$ & $2.94(2.78)$ \\
SU-n1 & 1 & $43.7(41.9)$ & $22.6(22.5)$ & 0.57 & $1.68(1.68)$ & $2.17(2.05)$ \\
SU-n10 & 10 & $12.2(10.6)$ & $8.56(8.34)$ & 1.04 & $0.92(0.85)$ & $1.60(1.43)$ \\
SU-n100 & 100 & $3.43(2.63)$ & $3.25(3.03)$ & 1.90 & $0.50(0.41)$ & $1.18(0.97)$ \\
\hline
\end{tabular}

Note. - Column 1: model name. Column 2: ambient medium hydrogen number density. Column 3: theoretical estimate of shell formation time $t_{\mathrm{sf}}$ (Equation (7)) and numerical measure $t_{\mathrm{sf}}^{\mathrm{n}}$ (in parentheses). Columns 4-7: theoretical estimates and numerical measures (in parentheses) of radius, postshock temperature, swept-up mass, and total momentum at shell formation.

Table 2. Physical Quantities at the Shell Formation for S2P Models

\begin{tabular}{|c|c|c|c|c|c|c|c|c|c|}
\hline Model & & $\begin{array}{c}\bar{n}_{H} \\
{\left[\mathrm{~cm}^{-3}\right]}\end{array}$ & $f_{V}$ & $f_{M}$ & $\begin{array}{c}t_{\mathrm{sf}}\left(t_{\mathrm{sf}}^{\mathrm{n}}\right) \\
{[\mathrm{kyr}]}\end{array}$ & $\begin{array}{c}r_{\mathrm{sf}}\left(r_{\mathrm{sf}}^{\mathrm{n}}\right) \\
{[\mathrm{pc}]}\end{array}$ & $\begin{array}{c}T_{\mathrm{sf}} \\
{\left[10^{6} \mathrm{~K}\right]}\end{array}$ & $\begin{array}{l}M_{\mathrm{sf}}\left(M_{\mathrm{sf}}^{\mathrm{n}}\right) \\
{\left[10^{3} M_{\odot}\right]}\end{array}$ & $\begin{aligned} & p_{\mathrm{sf}}\left(p_{\mathrm{sf}}^{\mathrm{n}}\right) \\
{\left[10^{5}\right.} & M_{\odot} \mathrm{km} \mathrm{s}^{-1}\end{aligned}$ \\
\hline \multirow{3}{*}{$\mathrm{S} 2 \mathrm{P}-\mathrm{n} 0.1$} & WHOLE & 0.1 & $\ldots$ & $\ldots$ & $156(384)$ & $59.5(83.3)$ & 0.31 & $3.08(3.44)$ & $2.94(3.21)$ \\
\hline & CNM & 1.5 & 0.06 & 0.81 & 36 & 19 & 0.62 & 1.5 & 2.1 \\
\hline & WNM & 0.017 & 0.92 & 0.15 & 420 & 130 & 0.19 & 4.9 & 3.7 \\
\hline \multirow{3}{*}{$\mathrm{S} 2 \mathrm{P}-\mathrm{n} 1$} & WHOLE & 1 & $\ldots$ & $\cdots$ & $43.7(100)$ & $22.6(31.5)$ & 0.57 & $1.68(1.44)$ & $2.17(2.13)$ \\
\hline & CNM & 8.9 & 0.09 & 0.81 & 13 & 9.0 & 1.0 & 0.94 & 1.6 \\
\hline & WNM & 0.14 & 0.84 & 0.12 & 130 & 52 & 0.34 & 2.8 & 2.8 \\
\hline \multirow{3}{*}{$\mathrm{S} 2 \mathrm{P}-\mathrm{n} 10$} & WHOLE & 10 & $\ldots$ & $\cdots$ & $12.2(24.3)$ & $8.56(10.7)$ & 1.04 & $0.92(0.70)$ & $1.60(1.46)$ \\
\hline & CNM & 110 & 0.08 & 0.83 & 3.3 & 3.2 & 1.9 & 0.49 & 1.2 \\
\hline & WNM & 1.5 & 0.88 & 0.13 & 35 & 19 & 0.64 & 1.5 & 2.1 \\
\hline \multirow{3}{*}{ S2P-n100 } & WHOLE & 100 & $\ldots$ & $\ldots$ & $3.43(5.30)$ & $3.25(3.63)$ & 1.90 & $0.50(0.36)$ & $1.18(1.00)$ \\
\hline & CNM & 1300 & 0.07 & 0.82 & 0.85 & 1.1 & 3.7 & 0.26 & 0.85 \\
\hline & WNM & 17 & 0.91 & 0.15 & 9.2 & 6.9 & 1.2 & 0.80 & 1.5 \\
\hline
\end{tabular}

Note. - Column 1: model name. Columns 3-5: mean hydrogen number density (whole medium - top row, CNM middle row, WNM - lower row); volume and mass fractions measured at saturation of TI. Columns 6-10: shell formation predicted time, radius, postshock temperature, swept-up mass, and total momentum for propagation into a uniform medium with density $\bar{n}_{H}$. See Equations (7), (8), (10), (11), and (17), respectively. Numerical measures at $t_{\mathrm{sf}}^{\mathrm{n}}$ averaged over 10 realizations of each model are shown in parentheses in the "WHOLE" rows. 


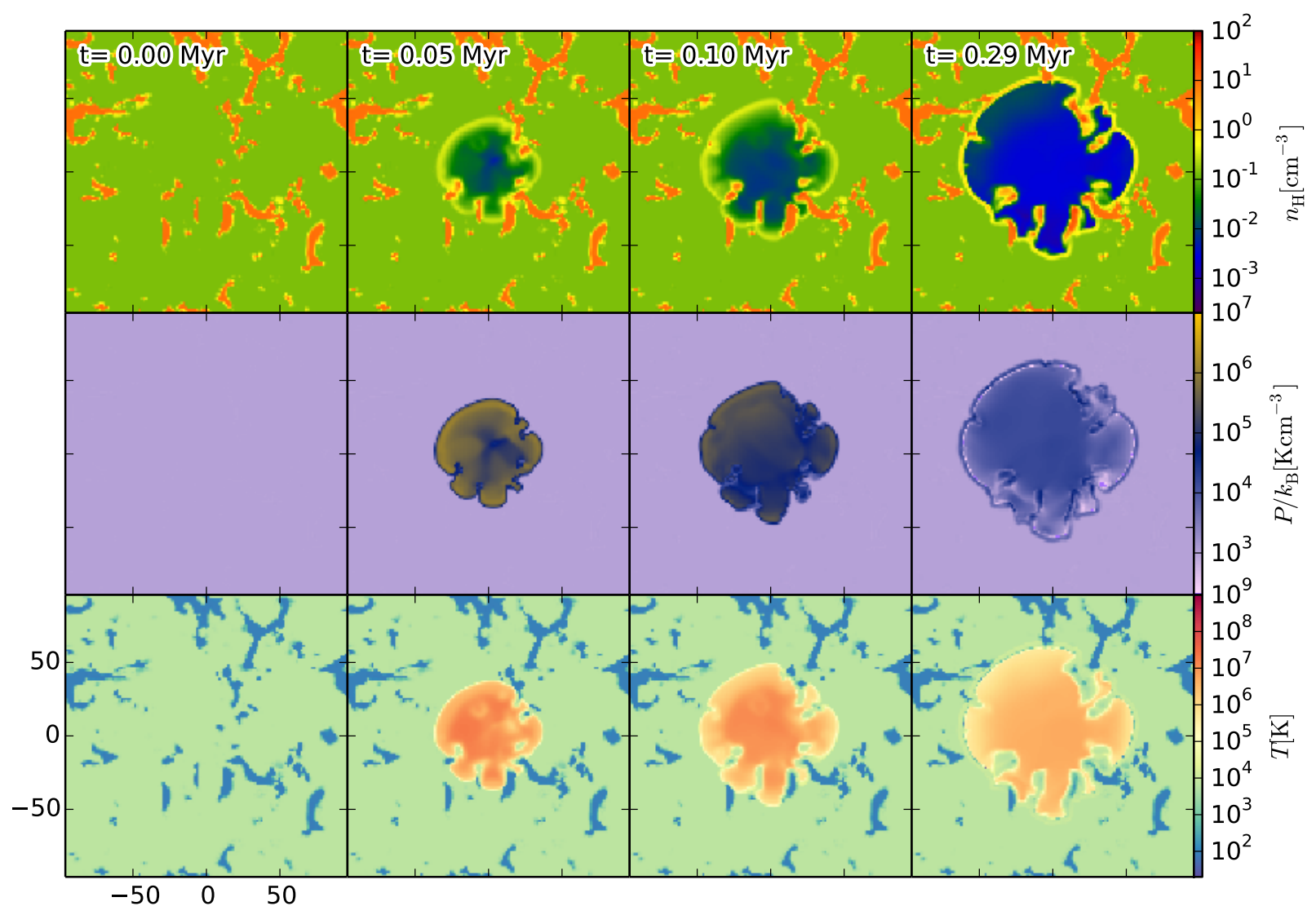

Fig. 9.- Example XY-slices of model S2P-n1 (two-phase initial medium with $n_{0}=1$ ) for grid resolution $\Delta=1.5$ pc. From top to bottom, logarithmic color scales show number density, pressure, and temperature. 

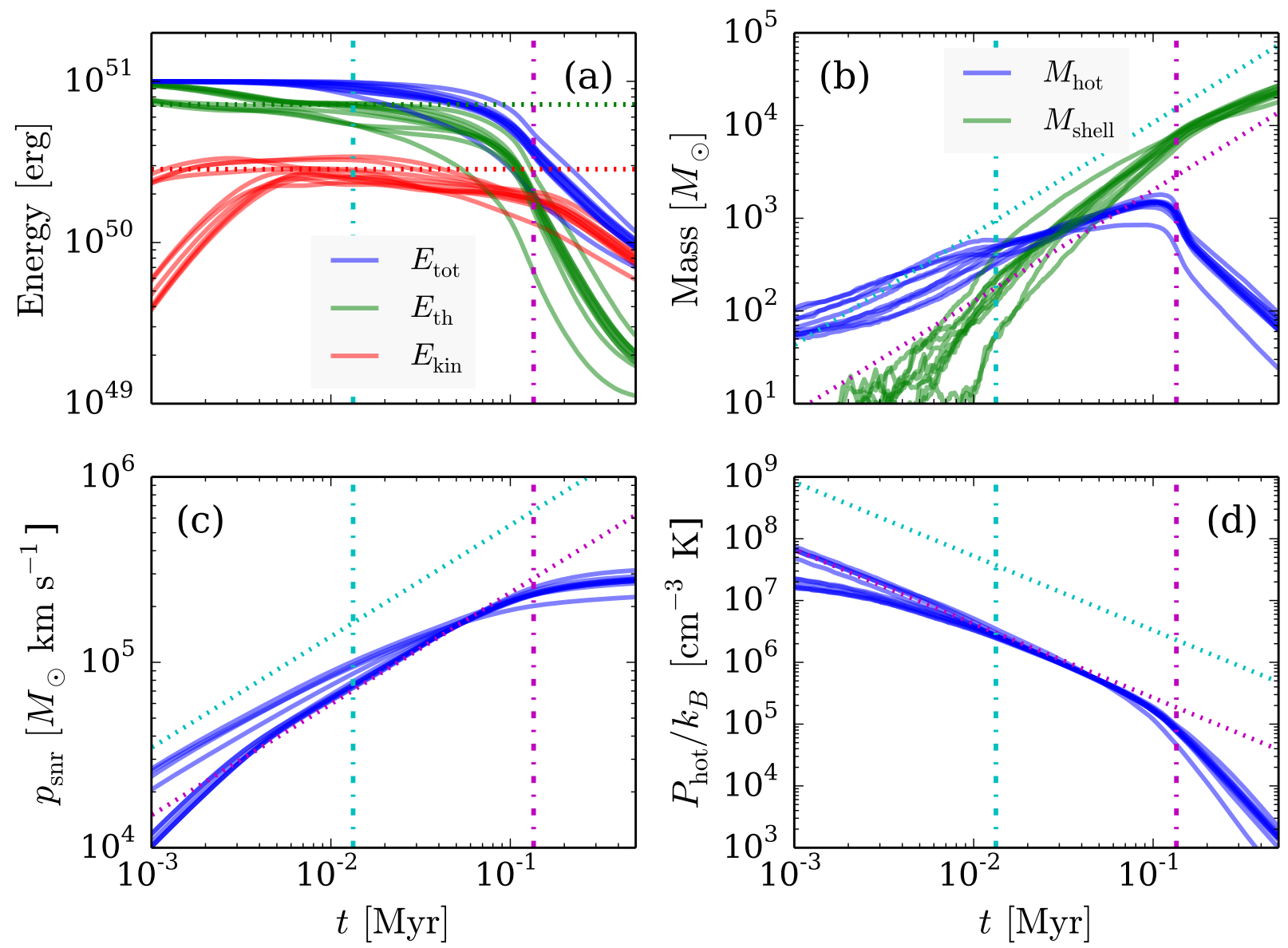

Fig. 10.- Time evolution for 10 different realizations of model S2P-n1. Shown are (a) total, thermal, and kinetic energies, (b) hot and shell gas masses, (c) total radial momentum, and (d) hot gas pressure. Vertical dot-dashed lines are the corresponding expected shell formation time for a uniform medium at the CNM (cyan) and WNM (magenta) density. Dotted lines are the ST solution for $n_{\mathrm{CNM}}(\mathrm{cyan})$ and $n_{\mathrm{WNM}}$ (magenta). 


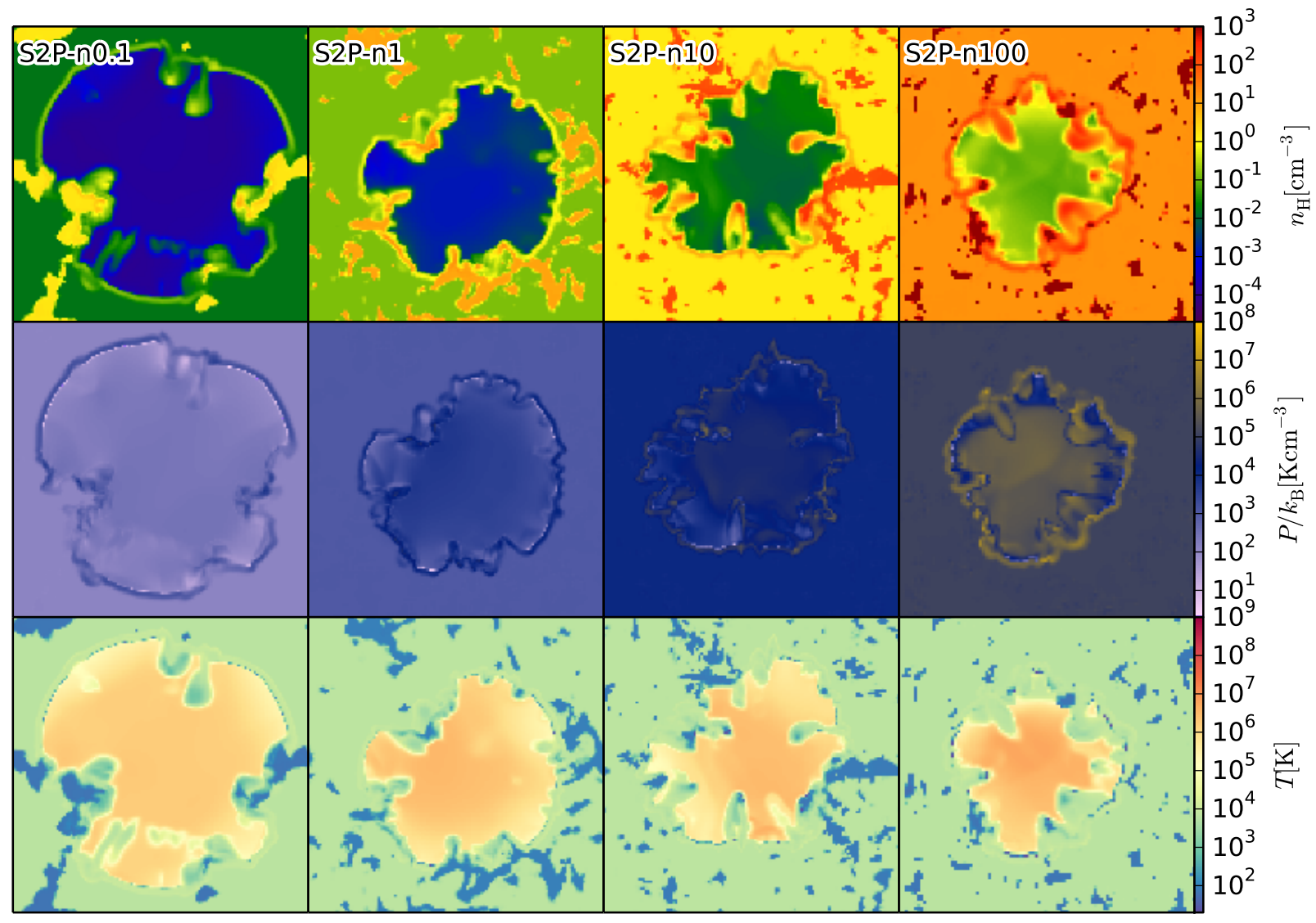

Fig. 11. - Example XY-slices of all S2P models at $t / t_{\mathrm{sf}} \sim 10$. The dimensions of the slices shown $384 \mathrm{pc} \times 384 \mathrm{pc}$ for S2P-n0.1, $192 \mathrm{pc} \times 192 \mathrm{pc}$ for S2P-n1, $64 \mathrm{pc} \times 64 \mathrm{pc}$ for S2P-n10, and $24 \mathrm{pc} \times 24 \mathrm{pc}$ for $\mathrm{S} 2 \mathrm{P}-\mathrm{n} 100$. The grid resolutions are $\Delta=3,1.5,0.5$, and $0.25 \mathrm{pc}$ for $n_{0}=0.1$, 1, 10, and 100. From top to bottom, logarithmic color scales show number density, pressure, and temperature. 

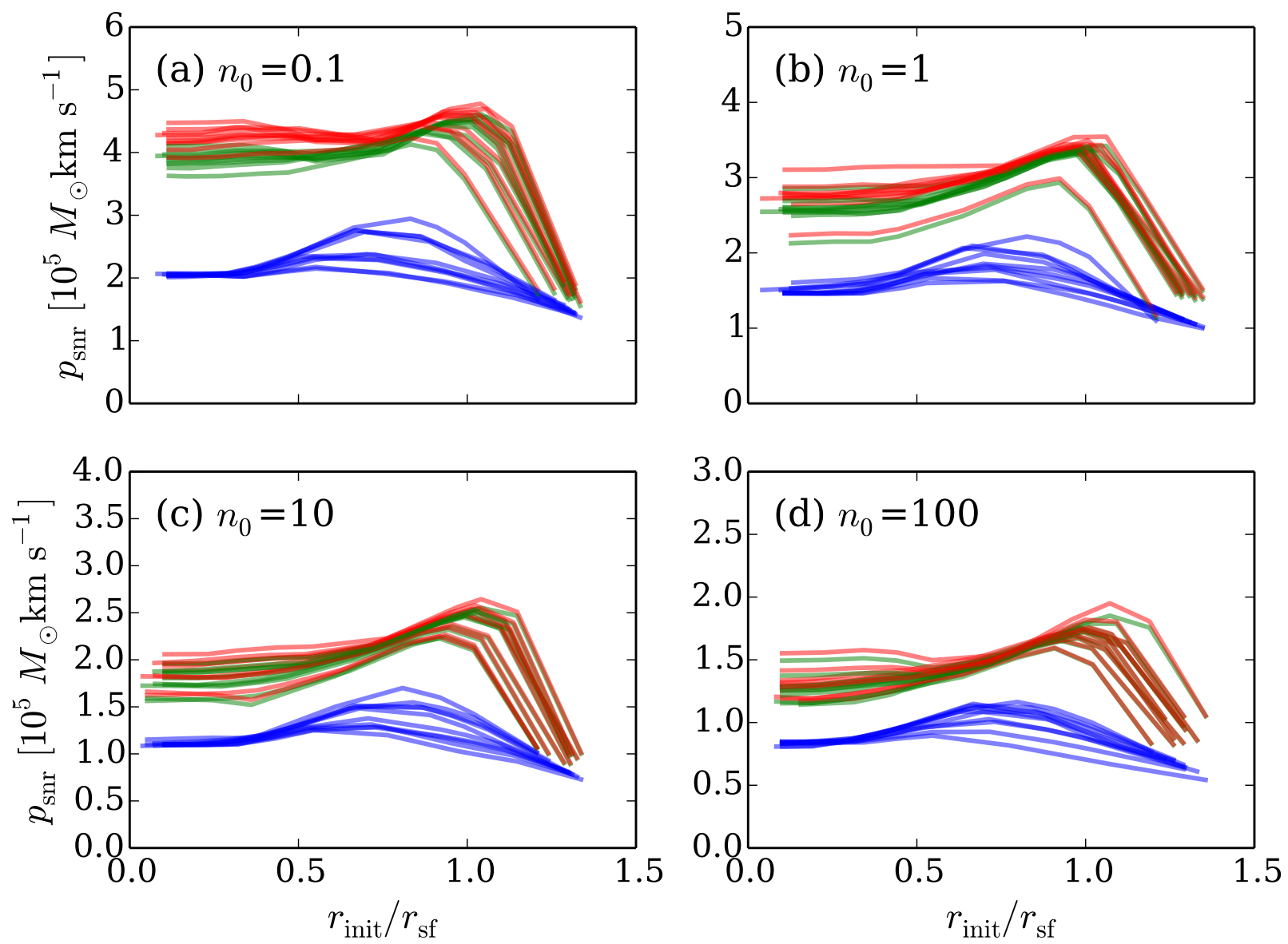

Fig. 12.- Convergence plots of total momentum as a function of the normalized initial radius of the SNR for S2P at $t / t_{\mathrm{sf}}=1$ (blue), 5 (green), 10 (red). The same condition derived from uniform background $\left(r_{\text {init }} / r_{\mathrm{sf}}<1 / 3\right.$; Section 4.2$)$ can be applied here for "consistent convergence". 
and mass fractions of the two phases are similar at all $n_{0}$, and the densities in each phase are proportional to $n_{0}$. Table 2 also lists for reference the physical quantities at shell formation that would apply for the ST solution propagating into uniform high-density (cold cloud) gas, uniform low density (intercloud) gas, and gas at the average density of the simulation. Numerical measures of the quantities averaged over 10 realizations are also shown in parentheses. As before, we define $t_{\mathrm{sf}}^{\mathrm{n}}$ as the time when the hot gas mass reaches a maximum.

Power-law fits for averaged numerical measures are given by

$$
\begin{gathered}
t_{\mathrm{sf}}^{\mathrm{n}}=9.6 \times 10^{4} \text { yr } n_{0}^{-0.62}, \\
r_{\mathrm{sf}}^{\mathrm{n}}=30.2 \mathrm{pc} n_{0}^{-0.46}, \\
M_{\mathrm{sf}, \mathrm{hot}}^{\mathrm{n}}=1540 M_{\odot} n_{0}^{-0.33},
\end{gathered}
$$

and

$$
p_{\mathrm{sf}}^{\mathrm{n}}=2.16 \times 10^{5} M_{\odot} \mathrm{km} \mathrm{s}^{-1} n_{0}^{-0.17} .
$$

Note that the above is the mass of hot gas at $t_{\mathrm{sf}}^{\mathrm{n}}$; there is several times as much "shell" gas because initially-cold, dense clouds are accelerated by the shock and by the expanding gas in the interior of the SNR. Compared to the uniform-medium case (Equations (22)-(25)), dependencies on the mean ambient density are similar. Although the shell formation epoch $t_{\mathrm{sf}}^{\mathrm{n}}$ is delayed for a twophase medium, the hot gas mass and total momentum at shell formation agree very well with the numerical measures of these quantities for a SNR in a uniform background (SU models), as well as analytic estimates.

Figure 9 displays snapshots in the XY plane of an example run for model S2P-n1. The time marked in the top row is the time since the explosion. For this run, we set the grid resolution to $\Delta=1.5 \mathrm{pc}$ and the initial size of SN to be $r_{\text {init }} / r_{\mathrm{sf}} \sim 0.2$, for an $r_{\mathrm{sf}}$ estimated using the mean density. For this specific example, the saturated two-phase ISM is characterized by $\left(n_{H}, T\right)=$ $\left(8.8 \mathrm{~cm}^{-3}, 106 \mathrm{~K}\right)$ and $\left(0.14 \mathrm{~cm}^{-3}, 6760 \mathrm{~K}\right)$ for CNM and WNM, respectively. The simulation volume is mainly occupied by the WNM with $f_{V}=0.84$, while the majority fraction $f_{M}=0.81$ of the mass resides in the CNM. Since the SNR expands solely through the WNM in most directions, the overall size of SNR is similar to expectations for a uniform medium with $n_{0}=0.14$ (especially in the 2nd quadrant of the XY plane in Figure 9). In some directions, the blastwave encounters small CNM clouds; these may be destroyed by the passage of the shock and/or form pillars within the SNR 7 Large CNM clouds are also shock compressed, and may partly block the SNR expansion, but over time they are also carried outward. At late times, the shell morphology is mainly shaped not by the shell instability observed in Figure 3 but by the initial structure of the ambient two-phase medium.

\footnotetext{
7 The ablation of clouds by dynamical instabilities is limited because of insufficient resolution. In addition, dense clouds may also evaporate after the shock passes if conduction from the surrounding hot medium is large enough; the present simulations do not include explicit conduction. Both effects are not fully captured, but see discussion in Section 7
} 
For S2P-n1 (10 different realizations), Figure 10 plots time evolution of (a) total, thermal, and kinetic energies, (b) hot and shell gas masses, (c) radial momentum, and (d) hot gas pressure. Also indicated with vertical dot-dashed lines are the ST solutions for the shell formation time when propagating into the CNM density (cyan; $t_{\mathrm{sf}, \mathrm{c}}$ ) and WNM density (magenta; $t_{\mathrm{sf}, \mathrm{w}}$ ). Dotted lines in panels (b), (c), (d) give ST solutions for an ambient medium that is 100\% CNM (cyan) and $100 \%$ WNM (magenta). Beginning at $\sim t_{\mathrm{sf}, \mathrm{c}}$, part of the SNR's energy is radiated away as the blastwave envelopes and shocks CNM clouds; both thermal and kinetic energy are reduced below the ST values. Since the most of volume is filled with the WNM, however, all physical quantities except the mass remain close to those of the ST solution in a uniform WNM until $\sim t_{\mathrm{sf}, \mathrm{w}}$. If there were no PDS stage, the momentum injected to a two-phase medium would be expected to lie between $p_{\text {sf }}$ for $\bar{n}_{\mathrm{CNM}}$ and $\bar{n}_{\mathrm{WNM}}$ (last column of Table 2). As $p_{\mathrm{sf}}$ is insensitive to the ambient medium density $\left(p_{\mathrm{sf}} \propto n_{0}^{-0.13}\right.$ predicted from Equation (7), or $p_{\mathrm{sf}} \propto n_{0}^{-0.17}$ using the numerical results in Table 1), and the momentum only modestly increases during the PDS stage, the total injected momentum by a single $\mathrm{SN}$ will always be of order $p_{\mathrm{sf}}$ for the mean density of the medium, $n_{0}$.

Figure 11 displays example XY-slices for S2P models with different mean density at $t / t_{\mathrm{sf}} \sim 10$. Although the initial cold and warm volume fractions are similar for all models, the cloud structures are different; there are more small clouds in higher density models. For the present models, the difference in structure at TI saturation is caused by differences in TI growth rates; smaller clouds can grow faster with higher heating rates in higher density models 8 The effective contribution from dense clouds increases with background density because of the increasing total cloud cross-section when there are more clouds. Thus, the numerical measures of physical quantities (in parentheses of Table 2) are closer to the analytic estimates for CNM as the mean density gets higher. However, the total injected momentum remains similar to the estimates from the mean density.

For ten different realizations at each mean density $n_{0}$, Figure 12 plots the radial momentum at $t / t_{\mathrm{sf}}=1$ (blue), 5 (green), and 10 (red), where $t_{\mathrm{sf}}$ is computed using $n_{0}$. Similar to Figure 6 . we show the result of varying the initial radius of the SNR. At each $n_{0}$, we use a reference value of $r_{\text {sf }}$ based on Equation (8) using $n_{0}$ (note, however, that the actual mean density within this $r_{\text {sf }}$ will differ from $n_{0}$ due to the particular realization of the cloudy structure). The resolution $\Delta$ is set to be smaller than $r_{\text {sf }} / 10$, which we have found (using tests similar to those of section 4.2 is sufficient for convergence. The horizontal axis is normalized by radius $r_{\mathrm{sf}}$ using $n_{0}$ (see Equation (8)). The final momentum converges provided $r_{\text {init }}<r_{\text {sf }} / 3$, similarly to what we found in the uniform medium case. This "consistent convergence" criterion guarantees convergence at all evolutionary stages; convergence at late stages only is possible up to slightly larger $r_{\text {init }}$. For $r_{\text {init }}>r_{\text {sf }}$, the final momentum is significantly lower than the converged value.

For mean number density of $n_{0}=0.1,1,10$, and 100, the mean values of the momentum at $t / t_{\mathrm{sf}}^{\mathrm{n}}=10$ are $p_{\text {final }} / p_{\mathrm{sf}}^{\mathrm{n}}=1.33,1.30,1.29$, and 1.32 , respectively. Similarly to Equation $(29)$, the

\footnotetext{
8 Additional physical ingredients ignored here such as turbulence, thermal conduction, and magnetic fields may also play an important role in shaping the ISM structure and in particular the size spectrum of cold clouds.
} 
final momentum in the physical units can be fitted by

$$
p_{\text {final }}=2.8 \times 10^{5} M_{\odot} \mathrm{km} \mathrm{s}^{-1} n_{0}^{-0.17} .
$$

The final momentum is always of order of $p_{\text {sf }}$, but slightly smaller for a clumpy medium than in the uniform-medium case. For different realizations of the model at each mean density (i.e. different seeds for the random perturbations), there are only $20-40 \%$ variations in the final momentum.

\section{Multiple SNe in Two-Phase Medium}

In Sections 4.1, 4.2, and 5, we considered the expansion of a single SNR in either a uniform or a clumpy medium for a wide range of mean densities $\left(n_{0}=0.1-100 \mathrm{~cm}^{-3}\right)$, covering conditions that would apply in the atomic or molecular ISM. There, we evaluated the momentum, mass of hot gas, and other basic properties of the SNR, and determined numerical requirements for consistent convergence, i.e. criteria such that simulation results agree with high-resolution benchmarks, at times before and after the SNR becomes strongly radiative. In this section, we extend to the case of multiple SNe that sequentially explode and expand into a surrounding two-phase medium. For multiple SNe, many more defining parameters enter the problem setup than for the case of a single SN, so a comprehensive study will be deferred to future work. However, it is useful to explore a few simple cases to assess potential differences in the momentum injection per event, compared to that from a single SN. Of course, the momentum injection from multiple spatially correlated SNe is also subject to global effects, since the superbubble that forms can expand beyond the scale height of the surrounding ISM and break out of the disk, venting into the galactic halo (e.g., Mac Low et al. 1989; Tenorio-Tagle et al. 1990; Hopkins et al. 2012a) and limiting further expansion within the ISM. Here, we do not consider these global effects (but see Section 7 for further discussion).

We consider the case in which SNe occur at intervals of 0.1Myr; this could represent the effects of a moderate-mass cluster $\left(M_{\text {cluster }} \sim 3 \times 10^{4} M_{\odot}\right)$ in which the event rate is not sufficient for energy deposition to reach the continuous limit. We consider a range of mean densities $n_{0}=1-10$ in the ambient medium, and as for the S2P models we create a "background" two-phase state by allowing the TI to reach saturation.

For the single-SN models, the initial size of the SNR was set relative to $r_{\text {sf }}$, the shell formation radius at the mean density of the ambient medium. Although for our tests $n_{0}$ has a pre-determined value and it is trivial to set $r_{\text {init }}$ directly, more generally the ambient density varies and it is more practical to define the initial SNR region based on its total enclosed mass. We take this approach for the M2P models. To obtain an equivalent condition to $r_{\text {init }} / r_{\text {sf }}<1 / 3$, we begin with $r_{\text {init }}=\Delta$ (thermal energy is distributed in eight zones closest to the center) and calculate the enclosed mass $M_{\text {init }} \equiv \sum_{r<r_{\text {init }}} \rho \Delta^{3}$ and the mean density $\rho_{0}=M_{\text {init }} / V_{\text {init }}$, where $V_{\text {init }}=\sum_{r<r_{\text {init }}} \Delta^{3}$. If $M_{\text {init }} / M_{\text {sf }}<1 / 27$ (where $M_{\text {sf }}$ is obtained with Equation (11) for this computed $\rho_{0}$ ) we increase $r_{\text {init }}$ by $\Delta / 2$ and repeat the calculation in order to get the largest possible initial size of SNR that is compatible with the convergence condition $r_{\text {init }} / r_{\text {sf }}<1 / 3$ (i.e. $M_{\text {init }} / M_{\text {sf }}<1 / 27$ ). 


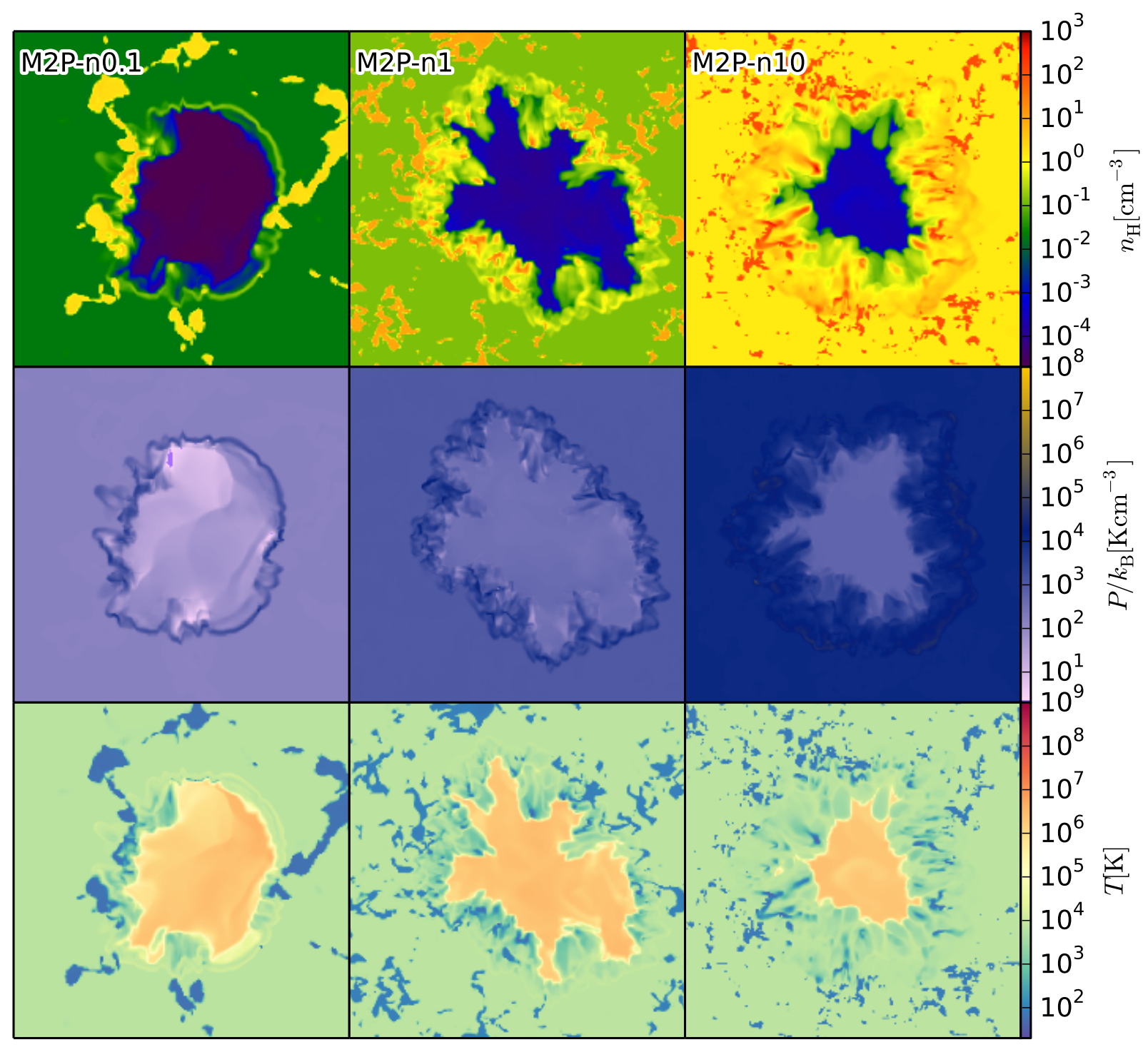

Fig. 13. - Example XY-slices of all M2P models at $t=2 \mathrm{Myr}$. The dimensions of the slices shown are $768 \mathrm{pc} \times 768 \mathrm{pc}$ for M2P-n0.1, $384 \mathrm{pc} \times 384 \mathrm{pc}$ for M2P-n1, and $192 \mathrm{pc} \times 192 \mathrm{pc}$ for M2P-n10, The grid resolutions are $\Delta=4,1.5$, and $0.75 \mathrm{pc}$ for $n_{0}=0.1,1$, and 10 . From top to bottom, logarithmic color scales show number density, pressure, and temperature. 

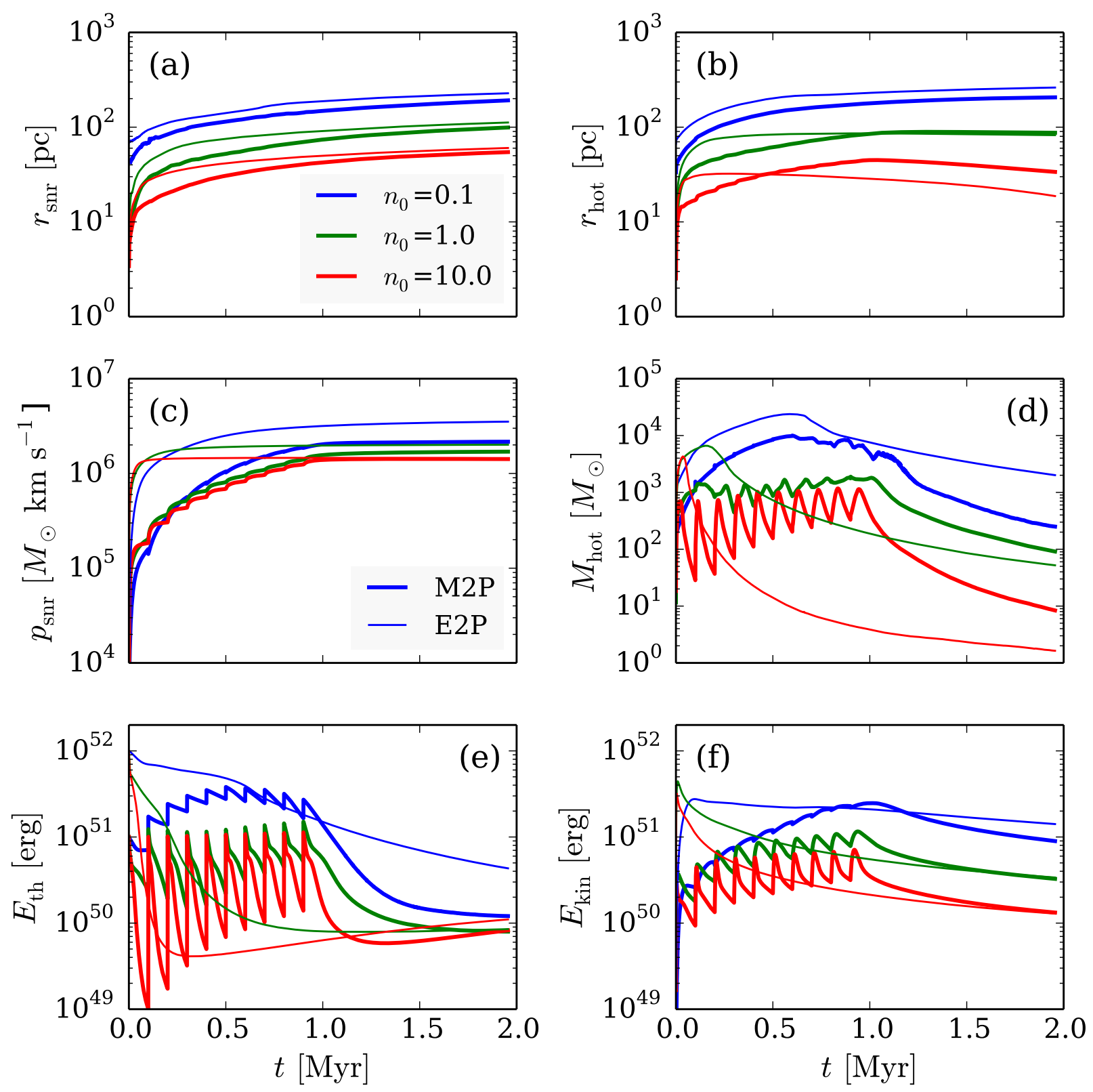

Fig. 14. - Time evolution of models with multiple SNe (M2P models, shown in thick lines), with $\mathrm{SN}$ interval interval $t_{\mathrm{SN}}=0.1 \mathrm{Myr}$ and $E_{\mathrm{SN}}=10^{51} \mathrm{erg}$. Panels show (a) the mass-weighted mean radius of the combined SNR $r_{\mathrm{snr}}$, (b) the effective radius of the hot gas volume $r_{\text {hot }} \equiv\left(3 V_{\text {hot }} / 4 \pi\right)^{1 / 3}$, (c) radial momentum, (d) hot gas mass, (e) thermal energy, and (f) kinetic energy. For comparison, single-event models $\left(\mathrm{E} 2 \mathrm{P}\right.$, with $\left.E_{\mathrm{SN}}=10^{52} \mathrm{erg}\right)$ are shown with thin lines. The mean density of the background ambient gas is $n_{0}=0.1$ (blue), 1 (green), and 10 (red). Multiple SNe become relatively less efficient at injecting momentum when the shell formation time is shorter, at higher ambient density. 
For each SN event, once a sphere with appropriate $r_{\text {init }}$ is defined, we redistribute the enclosed mass, momentum, and internal energy uniformly within $r_{\text {init }}$, and assign additional thermal energy adding up to $E_{\mathrm{SN}}$. This prescription prevents setting the temperature to extremely high values for very rarefied regions produced by previous SN explosions 9 All SNe explode at the center of the simulation domain.

For our M2P model set, we run each simulation until $10 \mathrm{SNe}$, with $E_{\mathrm{SN}}=10^{51}$ erg each, have exploded. For reference, we also run comparison simulations with a single explosion of energy $E_{\mathrm{SN}}=10^{52} \mathrm{erg}(\mathrm{E} 2 \mathrm{P})$. After the last $\mathrm{SN}$ (at $t=0.9 \mathrm{Myr}$ ), we run each simulation out until $t=2$ Myr to measure the final injected momentum.

Figure 13 displays XY-slices of the M2P models at final stage of evolution. The morphology of the SN bubble is determined by the structure of the ambient medium. Due to the short cooling time in model M2P-n10, the hot gas persists only deep inside of the SN bubble; this is in contrast to model M2P-n0.1, where the shell is still thin and the hot gas fills up the interior of the SN bubble. Figure 14 plots time evolution of (a) the mean radius of the combined SNR $r_{\mathrm{snr}}$, (b) the effective radius of hot gas volume $r_{\text {hot }} \equiv\left(3 V_{\text {hot }} / 4 \pi\right)^{1 / 3}$, (c) momentum $p_{\text {snr }}$, (d) hot gas mass $M_{\text {hot }}$, (e) thermal energy $E_{\mathrm{th}}$, and (f) kinetic energy $E_{\text {kin }}$. Thick lines show M2P and thin lines E2P models, with mean ambient density $n_{0}=0.1$ (blue), 1 (green), and 10 (red). Note that $r_{\mathrm{snr}}<r_{\text {hot }}$ at early stages when the SNR is filled with hot gas since the mass-weighted radius is closer to the shell radius in the CNM, and $r_{\mathrm{snr}}>r_{\text {hot }}$ at later stages when the hot gas is depleted (see right column in Figure 13).

Evidently, multiple SNe are able to inject more momentum when the ambient density is lower, similar to the case of single SNe. However, the dependence on ambient density is even weaker than in the single SN case. The final momenta for $n_{0}=0.1,1$, and 10 are $p_{\text {final }}=2.2,1.7$, and $1.4 \times 10^{6} M_{\odot} \mathrm{km} \mathrm{s}^{-1}$, respectively. There is negligible increase in momentum shortly after the final SN event, for all cases. Comparing multiple SNe to a single SN with the same total energy, the ratios of the total momentum from M2P models to E2P models are $61 \%, 85 \%$, and $98 \%$ for $n_{0}=0.1,1$, and 10, respectively. The final momentum per SN event fitted to these results is $1.7 \times 10^{5} M_{\odot} \mathrm{km} \mathrm{s}^{-1} n_{0}^{-0.1}$.

The differences among the M2P models can be understood partly based on the ratio of the SN time interval $t_{\mathrm{SN}}$ to the shell formation time $t_{\mathrm{sf}}$ for the ambient medium. The interval $t_{\mathrm{SN}}=0.1 \mathrm{Myr}$ corresponds to $t_{\mathrm{sf}}>t_{\mathrm{SN}}, t_{\mathrm{sf}} \sim t_{\mathrm{SN}}$, and $t_{\mathrm{sf}}<t_{\mathrm{SN}}$, for $n_{0}=0.1,1$, and 10 , respectively. For M2Pn10, new SN energy is injected only after completely exhausting the previous SN, since the interval

\footnotetext{
${ }^{9} \mathrm{~A}$ more realistic prescription, starting from a free expansion phase with ejecta, would be possible for subsequent SNe expanding into hot regions, because the first SN makes the ambient density low enough to resolve this phase on a grid that is suitable for large-scale ISM models. We defer explorations of this and other alternatives to future work, keeping in mind that complex "early" feedback such as stellar winds and H II region expansion may also alter the ambient state for secondary SNe. Here, we simply adopt a thermal energy initialization that is as similar as possible to our treatment of single SNR, in order to compare the momentum that is injected.
} 
is much longer than the shell formation time $t_{\mathrm{sf}}=0.012 \mathrm{Myr}$ in the ambient medium. The injection of momentum is thus in discrete events, and the mass of hot gas never reaches values much larger than that from a single SN event with $E_{\mathrm{SN}}=10^{51} \mathrm{erg}$. Model E2P-n10 has a much higher peak $M_{\text {hot }}$ than that of M2P-n10, because $M_{\mathrm{sf}}$ is nearly linearly proportional to $E_{\mathrm{SN}}$ (see Equation (11)). However, $M_{\text {hot }}$ drops rapidly after the early shell formation for model E2P-n10. In model M2P-n10, hot gas is replenished after each new SN event, resulting in larger occupying volume than E2P-n10. Both mass and volume of hot gas decrease after the last SN due to depletion of hot gas by cooling, while the mean radius of the SNR keeps increasing.

For M2P-n0.1, the evolution is quite different from M2P-n10. The mass of hot gas reaches nearly an order of magnitude higher than for M2P-n10, and as the hot medium does not fully cool in between events, the momentum injection becomes almost continuous rather than discrete. The peak in $M_{\text {hot }}$ occurs at nearly the same time for M2P-n0.1 as for E2P-n0.1, although for both the hot gas is rapidly depleted after this time. The volume occupied by hot gas keeps increasing as $r_{\mathrm{snr}}$ for both models.

The evolution of model M2P-n1 is intermediate between that of M2P-n10 and M2P-n0.1. The mass of hot gas stays close to constant between the first and last SN, with only slight dips. The hot gas volumes for M2P-n1 and E2P-n1 converge with each other. The momentum acquisition for model M2P-n1 is therefore smoother than for model M2P-n10, and more discrete than for model M2P-n0.1.

\section{SUMMARY \& DISCUSSION}

Utilizing the hydrodynamic code Athena with optically thin radiative cooling, we simulate the expansion of radiative SNRs on three-dimensional Cartesian grids. For all models, each explosion is numerically initiated by deposition of $E_{\mathrm{SN}}=10^{51} \mathrm{erg}$ in the form of thermal energy within a volume of radius $r_{\text {init }}$. We consider three types of simulations: a single SN explosion in a uniform medium (SU models; Section 4); a single SN explosion in a two-phase medium (S2P models; Section 5); and multiple SN explosions in a two-phase medium (M2P models; Section 6). We cover a range of mean density $n_{0}=0.1-100$. For two-phase models, the cloud and intercloud densities are respectively an order of magnitude above and below $n_{0}$.

With the SU simulations, we first delineate the detailed evolution of radiative SNRs, in comparison to analytic solutions and previous one-dimensional (spherical) numerical simulations. Among other quantities, we evaluate the time, radius, mass, and momentum of the SNR at shell formation, and the final momentum of the expanding cooled shell. We then conduct a wide, systematic parameter search to ascertain numerical conditions for "consistent convergence" (convergence for all evolutionary stages) of SNR evolution. We find that $r_{\text {init }} / r_{\text {sf }}<1 / 3$, where $r_{\text {sf }}$ the size of SNR at shell formation, guarantees consistent convergence, and the final momentum is within $25 \%$ or $18 \%$ of high-resolution benchmarks provided that the grid resolution is $\Delta / r_{\mathrm{sf}}<1 / 3$ or $\Delta / r_{\mathrm{sf}}<1 / 10$, 
respectively.

We next conduct similar studies using more realistic ambient conditions, consisting of a twophase ISM characterized by irregular CNM clouds embedded in the WNM. We show that the same numerical convergence conditions apply to the two-phase medium as the uniform medium, and evolution follows a similar course, with comparable hot gas mass and momentum injection. Finally, we investigate the momentum injection by multiple SNe in a two-phase medium, using the same numerical criteria to set the initial SNR size. The details of evolution depend on the interval between SNe compared to the shell formation time (which decreases with increasing mean ambient density), but the final momenta of the expanding (combined) SNRs are similar in all cases.

Our main findings and implications are summarized below.

\section{Stages of SNR Evolution -}

Our simulations confirm the well-known evolutionary stages of a SNR subsequent to early free expansion (e.g., Chevalier 1974; Cioffi et al. 1988; Thornton et al. 1998): an energy conserving Sedov-Taylor (ST) phase, shell formation when the remnant becomes radiative, a cooling-modified pressure-driven snowplow (PDS) phase, and a final momentum-conserving expansion phase. In a uniform medium, the shell formation epoch is well defined by the time at which the mass of hot gas attains its maximum, $\sim 10^{3} M_{\odot}$. Table 1 shows that the analytic estimate for $t_{\mathrm{sf}}$ from Equation (7) provides a reasonable match with our numericallymeasured $t_{\mathrm{sf}}^{\mathrm{n}}$; this also agrees with previous numerical findings for shell formation based on spherical one-dimensional simulations (Blondin et al. 1998; Thornton et al.1998). The radius, total SNR mass, and outward radial momentum at shell formation are also close to analytic estimates.

In classical theory (e.g., Ostriker \& McKee 1988; Draine 2011), an adiabatic PDS stage (with constant interior mass and $P_{\text {hot }} \propto r_{\text {snr }}^{-5}$ ) follows shell formation, but this ideal situation is not realized in practice (see also Cioffi et al. 1988, Thornton et al. 1998). Instead, the hot interior expands into the back of the shell, where it condenses and cools. The mass of hot gas and interior pressure drop by an order of magnitude within a few times $t_{\mathrm{sf}}$. With rapidlyfalling interior pressure, the momentum added to the shell during the PDS stage is modest (see below). Figure 4 shows that for a uniform medium, SNR evolution through ST, shell formation, cooling-modified PDS, and momentum-conserving stages is essentially congruent for varying ambient density, when normalized by the SNR properties at $t_{\mathrm{sf}}^{\mathrm{n}}$.

For a two-phase ambient medium, Figure 10 shows similar evolutionary stages to the case of a uniform medium. Although energy losses commence earlier (since dense clouds radiate after they are shock-compressed), the mass of hot gas reaches a maximum (and then rapidly declines) only when a strongly-cooling shell forms in the low-density volume-filling intercloud medium. The numerically-computed shell formation time is in between the values of $t_{\mathrm{sf}}$ computed using the mean ambient density and using the density of the intercloud medium 
(Table 2). A hot, low-density and low pressure bubble is left behind as dense cloudlets and an irregular shell of swept-up/cooled intercloud gas expand outward.

When multiple explosions occur sequentially in the same location, the evolution is either highly impulsive or relatively smooth depending on the interval between SNe compared to the shell formation time in the ambient medium (see Figure 14). Similar to the case of single $\mathrm{SNe}$, however, cooling of the interior implies that a constant asymptotic momentum is reached shortly after the last SN event.

Most of our simulations consider evolution of SNR in an unmagnetized medium. However, in the Appendix we include results for the evolution of magnetized models with a range of plasma beta parameter for the background medium ( $\beta \equiv P_{\mathrm{ISM}} / P_{\mathrm{mag}}$ in the range 0.1 to 10$)$. For $\beta=1$ and 10, evolution is essentially indistinguishable from the unmagnetized case up to about $10 t_{\mathrm{sf}}$. For the $\beta=0.1$ case, at late stages the shell thickens and becomes anisotropic due to magnetic pressure and tension forces, and at very late stages the SNR becomes elongated along the magnetic field (e.g., Hanayama \& Tomisaka 2006).

\section{Momentum Injection by a Single $S N-$}

Our simulations show that most of the momentum of the expanding remnant is acquired during the ST stage of evolution. For uniform ambient conditions, the final momentum is only $50 \%$ larger than the momentum $p_{\text {sf }}$ at shell formation, at all values of the ambient density (see Figure 4). With the very weak dependence of $p_{\text {sf }}$ on the ambient medium density (see Table (1) as predicted by Equation (17), the final momentum injected by a single SN in a uniform medium varies $\propto n_{0}^{-0.16}$, yielding $\sim(1-4) \times 10^{5} M_{\odot} \mathrm{km} \mathrm{s}^{-1}$ for $n_{0}=100-0.1$ (see Equation (29).

We show in Appendix that the momentum injection is the same for a magnetized medium as for the unmagnetized case, when the plasma beta parameter exceeds 0.1 . This is because most of the momentum is acquired during stages when thermal pressure within the SNR greatly exceeds magnetic forces.

For a two-phase ambient medium, most of the momentum is acquired during the period prior to shell formation in the low-density intercloud medium (Figure 10). For given mean density, there is some variation in when and how much total momentum is gained depending on the locations and sizes of dense clouds around the explosion site (see Figure 12), at a level of a few tens of percent. Averaging over different realizations, however, the momentum at the shell formation time (when the hot gas mass is maximal) is within $20 \%$ of the value predicted by Equation (17) using the mean density averaged over both dense clouds and low-density intercloud medium (see "WHOLE" rows in Table 2). After shell formation, the momentum increases by at most $50 \%$. For a cloudy medium, the final momentum from a single SN varies $\propto n_{0}^{-0.17}$ is also in the range $(1-4) \times 10^{5} M_{\odot} \mathrm{km} \mathrm{s}^{-1}$ when the $n_{0}=100-0.1$. (see Equation (34).

Our simulations show that the final momentum injected by a single SN is an order of mag- 
nitude larger than the initial momentum of the ejecta. This conclusion is insensitive to the mean density of the ambient medium, and irrespective of strong inhomogeneity in the ISM surrounding the explosion site. As a simple quantitative mnemonic, the momentum injected by a single $\mathrm{SN}$ is comparable to the initial energy of the blast $\left(10^{51} \mathrm{erg}\right)$ divided by the speed of the expanding shock wave when it becomes radiative $\left(\approx 200 \mathrm{~km} \mathrm{~s}^{-1}\right)$.

\section{Implications for SN Momentum Injection in the ISM -}

For scales larger than an individual star forming region, the amount of momentum injection to the ISM by a SN dominates other possible sources of feedback associated with earlier stages of a massive star's evolution, such as stellar winds, radiation pressure, and H II region expansion (see e.g., Matzner 2002; Murray et al. 2010; Ostriker \& Shetty 2011, for analytic estimates of inputs from these sources). In recent work, a simple momentum injection model (at a level $p_{\text {final }}=3 \times 10^{5} M_{\odot} \mathrm{km} \mathrm{s}^{-1}$ comparable to that found here) has been adopted to treat SN feedback, driving turbulence in the ISM. Simulations implementing this "pure momentum" approach have been used to model molecule-dominated starburst regions (Shetty \& Ostriker 2012), multiphase atomic-dominated regions (Kim et al. 2011, 2013), and barred spiral galaxies (Seo \& Kim 2013, 2014). Among the successes of these models are reaching realistic turbulence levels in the diffuse ISM, and achieving states of self-regulated star formation with rates that are consistent with observations (see also Ostriker et al. 2010; Ostriker \& Shetty 2011). In addition, Kim et al. (2014) demonstrated that synthetic H I $21 \mathrm{~cm}$ lines reconstructed from the models of Kim et al. (2013) are consistent with the characteristics of real $21 \mathrm{~cm}$ line observations by Roy et al. (2013b, see also Stanimirović et al. 2014).

In many recent investigations studying individual galaxies (e.g., Hopkins et al. 2012b; Teyssier et al. 2013; Agertz et al. 2013) or galaxy formation in a cosmological context (e.g., Guedes et al. 2011; Stinson et al. 2013; Hopkins et al. 2014), it has been found that models with only $\mathrm{SN}$ feedback are unable to limit star formation rates to realistic values. Concerns about the inefficacy of SNe has prompted increased attention to other modes of feedback, a valuable development in itself (especially for understanding the evolution of molecular clouds at stages prior to the first SN). However, these concerns are to some extent misplaced, and reflect numerical rather than physical limitations. In particular, it can be difficult to capture the crucial (but spatially small scale) ST stage of evolution - when most of the momentum is imparted - in simulations that must follow extremely large spatial scales and cannot afford high resolution for the ISM. In item 4 below, we summarize the numerical requirements for properly resolving SN momentum injection.

One reason for the current uncertainty about feedback from SN is that the real ISM is far from homogeneous, whereas previous focused studies of SNR expansion have mostly considered homogeneous (or at least isotropic) environments. For example, Agertz et al. (2013) applied only the initial ejecta momentum $p_{\text {free }}$ rather than the momentum at the end of ST phase $p_{\text {sf }}$ or the final momentum in the momentum conserving phase $p_{\text {final }}$, citing concerns regarding inhomogeneity of the site of SN explosion. However, as seen in Section 5 , inhomogeneity of the 
background medium does not significantly affect the total momentum injection compared to the uniform-medium case. The effective spatial scale for momentum deposition is larger in an inhomogeneous medium than in a uniform medium, however, because the shock propagates further in low-density intercloud gas before it becomes radiative.

Several independent studies contemporaneous with our own have also studied momentum injection by SNR in the inhomogeneous ISM. Although different groups have considered different environments and physical ingredients in their simulations, all have reached similar conclusions: the final injected momentum in a homogeneous and inhomogeneous medium are comparable to each other, and in good agreement with the results of our simulations. This result is irrespective of initialization methods to impose inhomogeneity; we begin with a two-phase cloud/intercloud medium produced by thermal instability, while Martizzi et al. (2014) adopt a lognormal density distribution, Walch \& Naab (2014) adopt a fractal density structure, and Iffrig \& Hennebelle (2014) initialize based on a turbulent velocity field. The latter two studies also consider SNe within radially stratified clouds. Walch \& Naab (2014) and Geen et al. (2014) find that explosion of the SN within a pre-existing low density H II region can enhance the momentum up to $\sim 5 \times 10^{5} M_{\odot} \mathrm{km} \mathrm{s}^{-1}$ (including the momentum injected by $\mathrm{H}$ II region expansion) for solar metallicity.

\section{Numerical Conditions for Correct SNR Evolution-}

At the resolution of current galaxy formation simulations (tens of parsecs or larger), and with realistic ISM densities, the ST phase cannot be properly resolved. This leads to the oft-cited "overcooling problem," namely that the energy of a SN is radiated away without having much impact on the ISM, if it is initially deposited in too large an initial volume or mass. Although this problem is well known, there has not previously been a systematic study to determine the numerical requirements needed to avoid it.

Here, we have conducted a large set of simulations varying both the grid resolution $(\Delta)$ and the initial size of the SNR $\left(r_{\text {init }}\right)$. We find that $r_{\text {init }} / r_{\text {sf }}<1 / 3$ is necessary to follow the hot gas evolution properly. We also find that the final momentum is within $18 \%$ and $25 \%$ of high-resolution benchmarks provided $\Delta<r_{\text {sf }} / 10$ and $r_{\text {sf }} / 3$, respectively. If the SN explosion occurs within an ambient medium density of $n_{0}=1$ or $10, r_{\mathrm{sf}}=23 \mathrm{pc}$ or $9 \mathrm{pc}$ thus gives a requirement of $\Delta \sim r_{\text {init }}<7$ pc or 3 pc, respectively, for $\Delta=r_{\text {init }}=r_{\text {sf }} / 3$

For practical purposes in an inhomogeneous medium, the resolution requirements can be expressed in terms of a maximum enclosed mass within the initial SNR. The criterion $r_{\text {init }} / r_{\text {sf }}<$ $1 / 3$, equivalent to $M_{\text {init }}<M_{\text {sf }} / 27$, corresponds to an enclosed mass of $M_{\text {init }}<(20-110) M_{\odot}$ for $n_{0}=100-0.1$. Interestingly, this is similar to the condition adopted in Joung \& Mac Low (2006), who argued that $M_{\text {init }} \sim 60 M_{\odot}$ would yield an initial temperature in the range of $10^{6}-10^{7} \mathrm{~K}$, for which the cooling rate would not be excessive. Joung \& Mac Low (2006) and subsequent studies by their colleagues (Joung et al. 2009; Hill et al. 2012) modelled local galactic disks with large vertical domains, including hot gas created by SN shocks. The numerical resolution of 1 pc combined with the condition $M_{\text {init }} \sim 60 M_{\odot}$ and dispersed SN 
events allowed them to correctly resolve the evolution of most SNRs. However, these models did not include self-gravity, which can lead to higher densities at the sites of SN events such that 1 pc grid resolution would be marginal or insufficient (considering that $r_{\mathrm{sf}} / 3 \sim 1 \mathrm{pc}$ for $\left.n_{0}=100\right)$; alternative approaches are discussed below.

\section{Alternative Feedback Prescriptions}

If ambient densities are not too high, and the grid is sufficiently fine, the needed resolution requirements $r_{\text {init }} / r_{\text {sf }}<1 / 3$ (or $M_{\text {init }}<M_{\text {sf }} / 27$ ) and $\Delta / r_{\text {sf }}<1 / 10$ can be met, and both the hot gas evolution and momentum injection of a SN can be properly followed by injecting thermal energy and allowing the SNR to progress through ST and radiative stages. We recommend this approach for best fidelity in modeling SN feedback. However, this is not always possible in numerical simulations, even with adaptive mesh refinement techniques.

In situations where it is impossible or impractical to resolve SNR evolution including the ST stage, an alternative is to inject momentum directly to the gas surrounding the feedback site. This will miss the direct effects of hot gas in the ISM (including potential losses of this gas as a galactic wind), but it will still drive turbulence in the warm and cold phases of the ISM, which enables star formation to be correctly self-regulated (Shetty \& Ostriker 2012; Kim et al. 2011, 2013). To allow for the (modest) dependence of momentum injection on ambient conditions, we recommend that Equation (34) be used to set the value of the momentum after the mean density in the numerical feedback region is measured.

In a spirit similar to the approach that we recommend, resolution-conditioned approaches to feedback in galaxy formation simulations have been implemented recently by Hopkins et al. (2014) and Kimm \& Cen (2014). In both studies, SN feedback is assigned either by the ejecta directly to nearby grid zones or SPH particles if the local resolution was high, or as "final" (post-ST) momentum otherwise. In their SPH simulations, Hopkins et al. (2014) compared the smoothing length with the shell formation radius $r_{\mathrm{sf}}\left(R_{\mathrm{cool}}\right.$ in their nomenclature $)$ derived by Cioffi et al. (1988) to determine which prescription to apply. In their AMR simulations, Kimm \& Cen (2014) apply either the initial ejecta momentum or a "final" (post-ST) momentum to zones depending on whether the mass in a given feedback sector is greater or less than a SNR would have at the "transition" time of Blondin et al. (1998). The resolution requirements we recommend, combined with our calibrations of momentum injection for varying mean density, may be useful in refining and further developing conditional-feedback prescriptions similar to these. The resolution requirements $r_{\text {init }} / r_{\mathrm{sf}}<1 / 3$ and $\Delta / r_{\mathrm{sf}}<1 / 10$ that we have obtained through convergence studies could be immediately applied in gridbased simulations that use similar finite-volume methods to those of the Athena code. For SPH simulations, it would be straightforward to conduct an analogous study to determine numerical requirements for convergence in the hot gas mass evolution and net momentum injection.

6. Multiple SNe and Superbubbles/Galactic Winds- 
When a sufficiently massive star cluster forms, one can expect not a single but multiple SN explosions to impact the surrounding ISM. Many previous studies of multiple SNe have focused on what requirements must be met for a superbubble to break out of the disk and drive a wind (e.g., Tomisaka \& Ikeuchi 1986; McCray \& Kafatos 1987; Mac Low et al. 1989 . Tenorio-Tagle et al. 1990; Koo \& McKee 1992a b; Roy et al. 2013a). In Section 6, we instead quantify momentum injection to the ISM, which is key to settling the turbulence level and rate of star formation. In this paper, we consider only a highly-idealized model of the impact of a moderate-sized star cluster on the surrounding ISM, in which SN blasts are intermittent. We explode $10 \mathrm{SNe}$ with time interval of $t_{\mathrm{SN}}=0.1 \mathrm{Myr}$ (M2P models) in a two-phase medium with density of $n_{0}=0.1,1$, and 10, following evolution for a total of $2 \mathrm{Myr}$ after the first explosion (and $1 \mathrm{Myr}$ after the last explosion). These specific conditions limit the SN bubble size smaller than or comparable to the disk scale height, allowing us to explore the momentum injection by a superbubble in a uniform medium.

When $t_{\mathrm{sf}}<t_{\mathrm{SN}}$, as for model M2P-n10, momentum is injected by individual events in a discrete fashion, whereas in the opposite limit of $t_{\mathrm{sf}}>t_{\mathrm{SN}}$ for model M2P-n0.1, momentum injection is almost continuous. In spite of these differences, there is only $\sim 50 \%$ variation in the final momentum measured in the three M2P models $\left(p_{\text {final }}=14-22 \times 10^{5} M_{\odot} \mathrm{km} \mathrm{s}^{-1}\right)$. As for single $\mathrm{SNe}$, the final momentum per $\mathrm{SN}$ event in the M2P models is comparable to the prediction $p_{\mathrm{sf}}$ of Equation (17). For comparison, we also conduct comparison models with a single SN having $E_{\mathrm{SN}}=10^{52}$ erg (E2P models); the final momenta for E2P models are a factor 1 - 2 larger than for the corresponding M2P models.

In recent one-dimensional simulations of sequential SNe, Sharma et al. (2014) have found that with a sufficiently large number of $\mathrm{SNe}$ (i.e. a sufficiently small mean interval $t_{\mathrm{SN}}$ ), the evolution of the SN bubble approaches the limit of continuous energy injection and an adiabatic interior, as in Weaver et al. (1977) (see also Tomisaka et al. 1981; McCray \& Kafatos 1987; Ostriker \& McKee 1988; Koo \& McKee 1992a b). For continuous energy injection, the radius evolves with $\eta=3 / 5$ rather than $\eta=2 / 5$ or $1 / 4$. Substantial energy (about $10-30 \%$ of total explosion energy) can remain in this limit (see also Vasiliev et al. 2015), as is required to explain observations of systems such as M82 (e.g., Strickland \& Heckman 2009).

Although our M2P models create large, low-density hot volumes that resemble observed superbubbles, here we have not considered a stratified medium or highly-dynamic initial conditions for the ambient warm/cold ISM. For a realistic cluster lifetime with $t_{\mathrm{SN}}=0.1 \mathrm{Myr}$, however, the total number of massive stars that could undergo core collapse is closer to 300, which would drive a superbubble expansion over a much longer period, such that its radius could easily exceed the disk scale height. More massive clusters would have even shorter mean intervals between SN events, and could easily lead to superbubble breakout. For more comprehensive studies for superbubble evolution, stratification of the disk should thus be taken into account, in addition to the small-scale inhomogeneity that we have included. A natural extension of the present work is to include both effects, together with multiple SNe, to study the superbubble breakout and creation of galactic winds that may occur when a very 
massive cluster forms.

Finally, we note some caveats related to limitations of the models we have presented. Because our resolution is insufficient to capture ablation of small clouds by the instabilities (Rayleigh-Taylor and Kelvin-Helmholtz) arising from shock-cloud interactions (e.g., Klein et al. 1994), our models may underestimate cooling within the SNR. Similarly, as we do not include thermal conduction (e.g., Cowie \& McKee 1977; Cowie et al. 1981; Balbus \& McKee 1982), we may be missing an increase in the mass of hot gas and enhanced cooling due to evaporation of and radiation from clouds. Compared to SNR evolution within a uniform intercloud medium, cloud ablation and evaporation lead to earlier shell formation as the effective density of the ambient medium is higher than that of the intercloud medium. The momentum injection could also be reduced somewhat due to additional radiative losses of energy. Although our simulation cannot fully capture cloud ablation and evaporation, these effects have been captured to some extent: in models S2P, the shell formation radius is smaller than that in a purely warm medium (see Table 2). Also, the final injected momentum is smaller in models S2P than SU (see Equations (29) and (34), and among 10 different realizations a slightly smaller momentum is obtained when the SNR sweeps up more cold clouds.

However, it is also important to note that effects of thermal conduction and cloud ablation become more complex for a magnetized medium, as is true for the ISM. Thermal conduction is anisotropic, so that the heat flux across the magnetic field is largely suppressed (Spitzer 1962). When a shock encounters a cloud, the magnetic field becomes wrapped around the cloud (piled up in front of the cloud, and trailing downstream). This geometry limits heat flux from the hot medium to the cloud. In addition, hydrodynamic instabilities are strongly suppressed with nearequipartition magnetic fields $\beta \sim 1$ (e.g., Mac Low et al. 1994; Fragile et al. 2005; Shin et al. 2008). Calculations of shock-cloud interactions including magnetic fields, anisotropic thermal conduction, and radiative cooling by Orlando et al. (2008) have shown that magnetic fields with moderate strength $(\beta=4)$ indeed suppress heat transfer and hydrodynamic instabilities (see also Johansson $\&$ Ziegler 2013). In future work, it will be interesting to extend the SNR expansion simulations of this paper to include magnetic fields and anisotropic conduction, in order to quantify possible changes in the momentum injection, maximum mass of hot gas, and other basic properties. In addition, complete models would include cosmic ray pressure, which can potentially boost the latetime SNR expansion and momentum. The magnitude of this effect depends on the diffusivity of the cosmic ray fluid, which is becoming increasing well characterized from recent hybrid kinetic simulations (e.g., Caprioli \& Spitkovsky 2014).

We are grateful to the referee for an extremely thorough report, which helped us to improve the manuscript. This work was supported by Grant No. AST-1312006 from the National Science Foundation. Part of this project was conducted during a visit to the KITP at U.C. Santa Barbara, which is supported by the National Science Foundation under Grant No. NSF PHY11-25915. This research made use of YT visualization and data analysis package (Turk et al. 2011). 


\section{REFERENCES}

Agertz, O., Kravtsov, A. V., Leitner, S. N., \& Gnedin, N. Y. 2013, ApJ, 770, 25

Aumer, M., White, S. D. M., Naab, T., \& Scannapieco, C. 2013, MNRAS, 434, 3142

Balbus, S. A., \& McKee, C. F. 1982, ApJ, 252, 529

Bandiera, R., \& Petruk, O. 2004, A\&A, 419, 419

Blondin, J. M., Wright, E. B., Borkowski, K. J., \& Reynolds, S. P. 1998, ApJ, 500, 342

Brook, C. B., Stinson, G., Gibson, B. K., Wadsley, J., \& Quinn, T. 2012, MNRAS, 424, 1275

Caprioli, D., \& Spitkovsky, A. 2014, ApJ, 794, 47

Chevalier, R. A. 1974, ApJ, 188, 501

Chevalier, R. A., \& Clegg, A. W. 1985, Nature, 317, 44

Chevalier, R. A., \& Gardner, J. 1974, ApJ, 192, 457

Cioffi, D. F., McKee, C. F., \& Bertschinger, E. 1988, ApJ, 334, 252

Cowie, L. L., \& McKee, C. F. 1977, ApJ, 211, 135

Cowie, L. L., McKee, C. F., \& Ostriker, J. P. 1981, ApJ, 247, 908

Cox, D. P. 1972, ApJ, 178, 159

-. 1986, ApJ, 304, 771

-. 2005, ARA\&A, 43, 337

Cox, D. P., \& Anderson, P. R. 1982, ApJ, 253, 268

Cox, D. P., \& Smith, B. W. 1974, ApJ, 189, L105

de Avillez, M. A., \& Breitschwerdt, D. 2004, A\&A, 425, 899

Dobbs, C. L., Krumholz, M. R., Ballesteros-Paredes, J., et al. 2013, ArXiv e-prints, arXiv:1312.3223

Draine, B. T. 2011, Physics of the Interstellar and Intergalactic Medium

Ferriere, K. 1998, ApJ, 503, 700

Ferrière, K. M. 2001, Reviews of Modern Physics, 73, 1031

Ferriere, K. M., Mac Low, M.-M., \& Zweibel, E. G. 1991, ApJ, 375, 239

Field, G. B. 1965, ApJ, 142, 531 
Field, G. B., Goldsmith, D. W., \& Habing, H. J. 1969, ApJ, 155, L149

Fragile, P. C., Anninos, P., Gustafson, K., \& Murray, S. D. 2005, ApJ, 619, 327

Geen, S., Rosdahl, J., Blaizot, J., Devriendt, J., \& Slyz, A. 2014, ArXiv e-prints, arXiv:1412.0484

Guedes, J., Callegari, S., Madau, P., \& Mayer, L. 2011, ApJ, 742, 76

Hanayama, H., \& Tomisaka, K. 2006, ApJ, 641, 905

Heiles, C., \& Troland, T. H. 2003, ApJ, 586, 1067

Hennebelle, P., \& Iffrig, O. 2014, A\&A, 570, A81

Hill, A. S., Joung, M. R., Mac Low, M.-M., et al. 2012, ApJ, 750, 104

Hopkins, P. F., Kereš, D., Oñorbe, J., et al. 2014, MNRAS, 445, 581

Hopkins, P. F., Quataert, E., \& Murray, N. 2012a, MNRAS, 421, 3522

-. 2012b, MNRAS, 421, 3488

Iffrig, O., \& Hennebelle, P. 2014, ArXiv e-prints, arXiv:1410.7972

Johansson, E. P. G., \& Ziegler, U. 2013, ApJ, 766, 45

Joung, M. K. R., \& Mac Low, M.-M. 2006, ApJ, 653, 1266

Joung, M. R., Mac Low, M.-M., \& Bryan, G. L. 2009, ApJ, 704, 137

Katz, N. 1992, ApJ, 391, 502

Keller, B. W., Wadsley, J., Benincasa, S. M., \& Couchman, H. M. P. 2014, MNRAS, 442, 3013

Kim, C.-G., Kim, W.-T., \& Ostriker, E. C. 2008, ApJ, 681, 1148

—. 2011, ApJ, 743, 25

Kim, C.-G., Ostriker, E. C., \& Kim, W.-T. 2013, ApJ, 776, 1

-. 2014, ApJ, 786, 64

Kimm, T., \& Cen, R. 2014, ApJ, 788, 121

Klein, R. I., McKee, C. F., \& Colella, P. 1994, ApJ, 420, 213

Könyves, V., Kiss, C., Moór, A., Kiss, Z. T., \& Tóth, L. V. 2007, A\&A, 463, 1227

Koo, B.-C., \& McKee, C. F. 1992a, ApJ, 388, 93

-. 1992b, ApJ, 388, 103 
Koyama, H., \& Inutsuka, S.-i. 2002, ApJ, 564, L97

Krumholz, M. R., Bate, M. R., Arce, H. G., et al. 2014, ArXiv e-prints, arXiv:1401.2473

Lemaster, M. N., \& Stone, J. M. 2009, ApJ, 691, 1092

Mac Low, M.-M., \& Klessen, R. S. 2004, Reviews of Modern Physics, 76, 125

Mac Low, M.-M., McCray, R., \& Norman, M. L. 1989, ApJ, 337, 141

Mac Low, M.-M., McKee, C. F., Klein, R. I., Stone, J. M., \& Norman, M. L. 1994, ApJ, 433, 757

Martizzi, D., Faucher-Giguère, C.-A., \& Quataert, E. 2014, ArXiv e-prints, arXiv:1409.4425

Matzner, C. D. 2002, ApJ, 566, 302

McCray, R., \& Kafatos, M. 1987, ApJ, 317, 190

McKee, C. F., \& Ostriker, E. C. 2007, ARA\&A, 45, 565

McKee, C. F., \& Ostriker, J. P. 1977, ApJ, 218, 148

Murray, N., Quataert, E., \& Thompson, T. A. 2010, ApJ, 709, 191

Nakamura, F., McKee, C. F., Klein, R. I., \& Fisher, R. T. 2006, ApJS, 164, 477

Norman, C. A., \& Ikeuchi, S. 1989, ApJ, 345, 372

Orlando, S., Bocchino, F., Reale, F., Peres, G., \& Pagano, P. 2008, ApJ, 678, 274

Ostriker, E. C., McKee, C. F., \& Leroy, A. K. 2010, ApJ, 721, 975

Ostriker, E. C., \& Shetty, R. 2011, ApJ, 731, 41

Ostriker, J. P., \& McKee, C. F. 1988, Reviews of Modern Physics, 60, 1

Piontek, R. A., \& Ostriker, E. C. 2004, ApJ, 601, 905

Rosen, A., \& Bregman, J. N. 1995, ApJ, 440, 634

Roy, A., Nath, B. B., Sharma, P., \& Shchekinov, Y. 2013a, MNRAS, 434, 3572

Roy, N., Kanekar, N., Braun, R., \& Chengalur, J. N. 2013b, MNRAS, 436, 2352

Sanders, R., Morano, E., \& Druguet, M.-C. 1998, Journal of Computational Physics, 145, 511

Scannapieco, C., Wadepuhl, M., Parry, O. H., et al. 2012, MNRAS, 423, 1726

Sedov, L. I. 1959, Similarity and Dimensional Methods in Mechanics

Seo, W.-Y., \& Kim, W.-T. 2013, ApJ, 769, 100 
-. 2014, ApJ, 792, 47

Sharma, P., Roy, A., Nath, B. B., \& Shchekinov, Y. 2014, MNRAS, 443, 3463

Shetty, R., \& Ostriker, E. C. 2012, ApJ, 754, 2

Shin, M.-S., Stone, J. M., \& Snyder, G. F. 2008, ApJ, 680, 336

Slavin, J. D., \& Cox, D. P. 1992, ApJ, 392, 131

Spitzer, L. 1962, Physics of Fully Ionized Gases

—. 1978, Physical processes in the interstellar medium

Springel, V., \& Hernquist, L. 2003, MNRAS, 339, 289

Stanimirović, S., Murray, C. E., Lee, M.-Y., Heiles, C., \& Miller, J. 2014, ApJ, 793, 132

Sternberg, A., Le Petit, F., Roueff, E., \& Le Bourlot, J. 2014, ApJ, 790, 10

Stinson, G., Seth, A., Katz, N., et al. 2006, MNRAS, 373, 1074

Stinson, G. S., Brook, C., Macciò, A. V., et al. 2013, MNRAS, 428, 129

Stone, J. M., \& Gardiner, T. 2009, New A, 14, 139

Stone, J. M., Gardiner, T. A., Teuben, P., Hawley, J. F., \& Simon, J. B. 2008, ApJS, 178, 137

Strickland, D. K., \& Heckman, T. M. 2009, ApJ, 697, 2030

Strickland, D. K., \& Stevens, I. R. 2000, MNRAS, 314, 511

Sutherland, R. S., \& Dopita, M. A. 1993, ApJS, 88, 253

Taylor, G. 1950, Royal Society of London Proceedings Series A, 201, 159

Tenorio-Tagle, G., Rozyczka, M., \& Bodenheimer, P. 1990, A\&A, 237, 207

Teyssier, R., Pontzen, A., Dubois, Y., \& Read, J. I. 2013, MNRAS, 429, 3068

Thacker, R. J., \& Couchman, H. M. P. 2000, ApJ, 545, 728

Thornton, K., Gaudlitz, M., Janka, H.-T., \& Steinmetz, M. 1998, ApJ, 500, 95

Tomisaka, K., Habe, A., \& Ikeuchi, S. 1981, Ap\&SS, 78, 273

Tomisaka, K., \& Ikeuchi, S. 1986, PASJ, 38, 697

Turk, M. J., Smith, B. D., Oishi, J. S., et al. 2011, ApJS, 192, 9

Vasiliev, E. O., Nath, B. B., \& Shchekinov, Y. 2015, MNRAS, 446, 1703 
Vishniac, E. T. 1983, ApJ, 274, 152

-. 1994, ApJ, 428, 186

Walch, S. K., \& Naab, T. 2014, ArXiv e-prints, arXiv:1410.0011

Weaver, R., McCray, R., Castor, J., Shapiro, P., \& Moore, R. 1977, ApJ, 218, 377

Wolff, M. T., \& Durisen, R. H. 1987, MNRAS, 224, 701

Wolfire, M. G., Hollenbach, D., McKee, C. F., Tielens, A. G. G. M., \& Bakes, E. L. O. 1995a, ApJ, 443,152

Wolfire, M. G., McKee, C. F., Hollenbach, D., \& Tielens, A. G. G. M. 1995b, ApJ, 453, 673

—. 2003, ApJ, 587, 278

Woltjer, L. 1972, ARA\&A, 10, 129

\section{A. Single SN in Uniform, Magnetized Medium}

The effect of magnetic fields in SNR evolution was initially investigated using spherically symmetric one-dimensional simulations, in which magnetic pressure forces are included but the full MHD equations are not solved (Chevalier 1974; Slavin \& Cox 1992) (this can approximately represent an equatorial band tangent to a uniform external magnetic field). The strong magnetic pressure within the compressed shell results in a thicker shell and recompresses hot gas in the later times. Hanayama \& Tomisaka (2006) followed the long-term evolution of a SNR in a magnetized medium using axisymmetric two-dimensional simulations, and showed that the magnetic tension force also helps broaden the shell and compress the hot gas (see also Ferriere et al. 1991, for supperbubble expansion). Magnetic fields play an important role in determining the volume of the hot gas in very late stages of a SNR, and therefore affects the porosity of the ISM.

Here, we are interested in assessing potential effects of magnetic fields on momentum injection by SNRs in the ISM. We have re-run the set of SU models, but now include a uniform magnetic field in the $z$-direction $\mathbf{B}_{0}=\left(0,0, B_{z, 0}\right)$. The magnetic field strength is set by using the plasma beta

$$
\beta \equiv \frac{8 \pi P_{\mathrm{ISM}}}{B_{z, 0}^{2}},
$$

where the thermal pressure of the ISM in our models varies with the mean density, $n_{0} \equiv n_{H} / 1 \mathrm{~cm}^{-3}$, as $P_{\mathrm{ISM}}=1500 n_{0} k_{\mathrm{B}} \mathrm{cm}^{-3} \mathrm{~K}$. For reference, the magnetic field strength is

$$
B_{z, 0}=3.2 \mu G\left(\frac{P_{\mathrm{ISM}} / k_{\mathrm{B}}}{3000 \mathrm{~cm}^{-3} \mathrm{~K}}\right)^{1 / 2} \beta^{-1 / 2}=2.3\left(n_{0} / \beta\right)^{1 / 2} \mu G .
$$

This preprint was prepared with the AAS LATEX macros v5.2. 

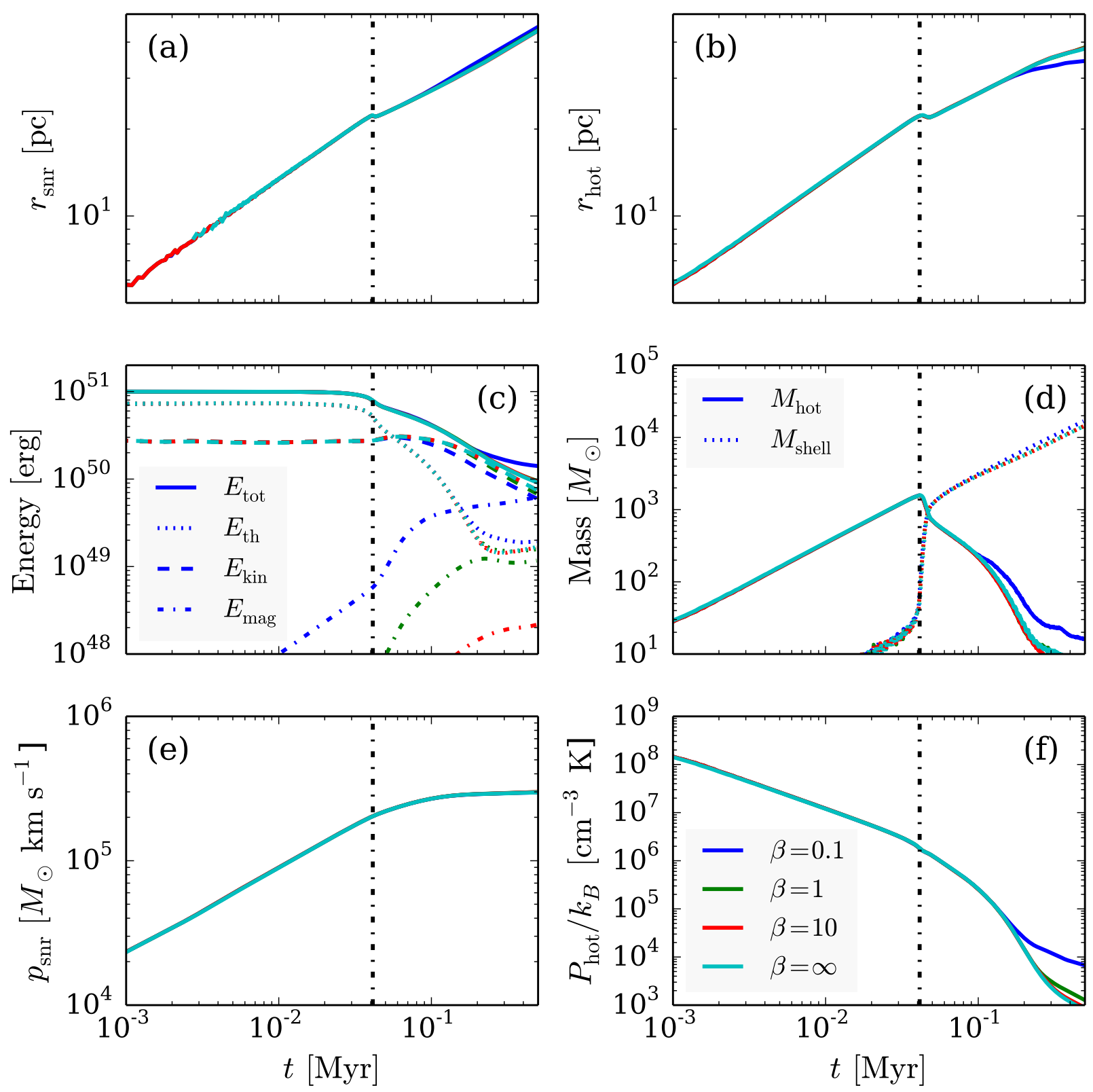

Fig. 15. - Time evolution of models analogous to SU-n1 $\left(n_{0}=1 \mathrm{~cm}^{-3}\right.$ and $\left.E_{\mathrm{SN}}=10^{51} \mathrm{erg}\right)$, but with initial uniform magnetic fields characterized by varying $\beta$. (a) mass-weighted radius, (b) the effective radius of the hot gas volume, (c) total, thermal and kinetic energies, (d) mass of interior hot gas and shell, (e) total radial momentum, and (f) pressure of interior hot gas. Grid resolution is $\Delta=1 / 2 \mathrm{pc}$ and the initial SNR radius is $r_{\text {init }}=3 \mathrm{pc}$. The vertical dot-dashed lines in each panel denote the predicted shell formation time $t_{\mathrm{sf}}=4.4 \times 10^{4} \mathrm{yr}$ (Equation (7)) for this model without magnetic fields. 


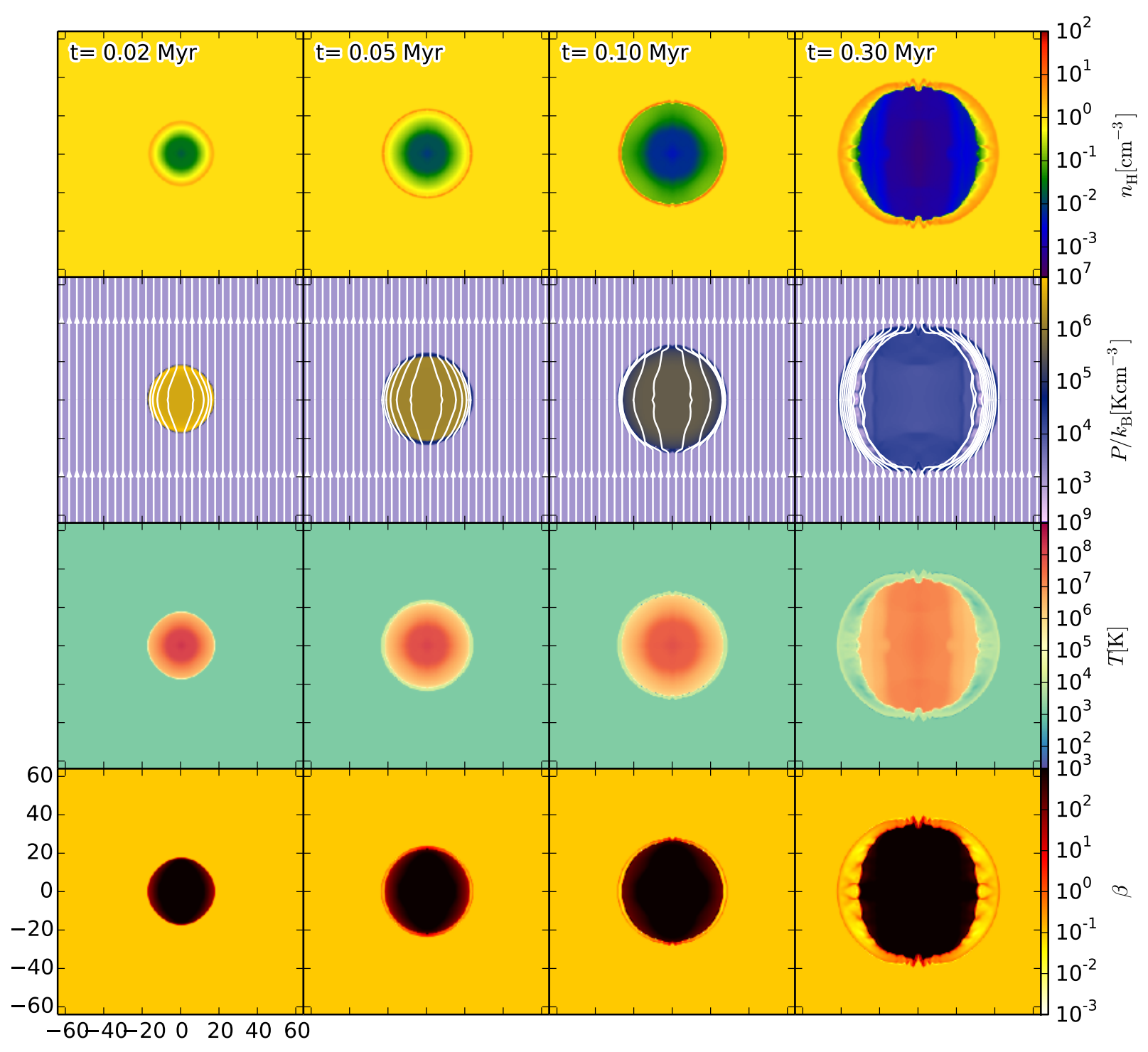

Fig. 16. - YZ-slices from magnetized model with $n_{0}=1$ and $\beta=0.1$, corresponding to $B_{z, 0}=$ $7.2 \mu G$. From top to bottom, logarithmic color scales show number density, pressure, temperature, and plasma beta. From left to right, columns correspond to snapshots at $t / t_{\mathrm{sf}} \sim 1 / 2,1,2$, and 7 . Magnetic field lines are shown in the second row (pressure). 
Figure 15 plots time evolution of physical properties of the SNR in models SU-n1 with $\beta=0.1$, 1,10 , and $\infty$. All models follow almost the same evolution except at late time in the model with $\beta=0.1$. It is obvious that the magnetic energy in the shell becomes comparable to the thermal energy of the shell only for $\beta=0.1$ after shell formation (see Figure 15(c)). As reported in earlier work, a strong magnetic field within the shell recompresses the interior hot gas (smaller $r_{\text {hot }}$ at late time for $\beta=0.1$ ), leading to larger $M_{\text {hot }}$ and $P_{\text {hot }}$ at late times (see Figure 15 (d) and (f)). However, the radial momentum is almost the same for all cases. This is because the majority of the momentum is injected to the ISM before the magnetic energy in the shell is very large. Even for the $\beta=0.1$ case, full momentum is acquired by $(2-3) t_{\mathrm{sf}}$, when the magnetic energy just begins to dominate the thermal energy.

Figure 16 displays the structure of the model with $n_{0}=1$ and $\beta=0.1$ in the YZ plane. Early evolution is the same as for the unmagnetized model (left three columns, see Figure 3), while nonspherical structure emerges in the latest stage (rightmost column). The outer shock can propagate farther in the direction perpendicular to the magnetic fields (fast mode), while shell broadening results in compression of the interior hot gas.

We have also performed simulations analogous to models SU-n0.1, SU-n10, and SU-n100 with $\beta=0.1,1$, and 10. We find that the normalized evolution for all cases is "congruent" (as in unmagnetized models) up to 2 or $3 t_{\mathrm{sf}}$, so that the final momentum remains unchanged. This suggests that ISM magnetic fields with reasonable strengths of $\beta=0.1-10$ may be able to alter late time evolution and porosity of the hot gas, but would not significantly affect the injected momentum (see also Iffrig \& Hennebelle 2014). 